\title{
Triangular-lattice anisotropic dimerized Heisenberg antiferromagnet: Stability and excitations of the quantum paramagnetic phase
}

\author{
R. L. Doretto \\ Instituto de Física Teórica, Universidade Estadual Paulista, 01140-070 São Paulo, SP, Brazil \\ Matthias Vojta \\ Institut für Theoretische Physik, Technische Universität Dresden, 01062 Dresden, Germany
}

(Received 22 November 2011; published 26 March 2012)

\begin{abstract}
Motivated by experiments on nonmagnetic triangular-lattice Mott insulators, we study one candidate paramagnetic phase, namely the columnar dimer (or valence-bond) phase. We apply variants of the bond-operator theory to a dimerized and spatially anisotropic spin- $1 / 2$ Heisenberg model and determine its zero-temperature phase diagram and the spectrum of elementary triplet excitations (triplons). Depending on model parameters, we find that the minimum of the triplon energy is located at either a commensurate or an incommensurate wave vector. Condensation of triplons at this commensurate-incommensurate transition defines a quantum Lifshitz point, with effective dimensional reduction that possibly leads to nontrivial paramagnetic (e.g., spin-liquid) states near the closing of the triplet gap. We also discuss the two-particle decay of high-energy triplons, and we comment on the relevance of our results for the organic Mott insulator $\mathrm{EtMe}_{3} \mathrm{P}\left[\mathrm{Pd}(\mathrm{dmit})_{2}\right]_{2}$.
\end{abstract}

DOI: 10.1103/PhysRevB.85.104416

\section{INTRODUCTION}

In the search for novel phases, frustrated quantum antiferromagnets have attracted an enormous amount of attention. Here, the combined effect of geometric frustration and quantum fluctuations tends to destabilize conventional magnetic order in favor of quantum paramagnetic ground states, such as valence-bond solids (VBS) with broken lattice symmetries or featureless spin liquids. ${ }^{1-3}$

A prominent example of a frustrated quantum magnet is the spin-1/2 Heisenberg model on the triangular lattice. Although it was initially proposed by Anderson ${ }^{4}$ that its ground state could be a resonating-valence-bond (RVB) spin liquid, it is now established that the model displays semiclassical noncollinear $120^{\circ}$ order. $^{5}$ However, modifications beyond the simple nearest-neighbor exchange are believed to induce nonmagnetic phases on the triangular lattice. For instance, it has been $\operatorname{argued}^{6}$ that a combination of longer-range and multiple-spin-exchange interactions is important near the Mott transition to a metallic state, where they induce a spin-liquid state as observed in numerical studies of the triangular-lattice Hubbard model. Models with spatially anisotropic exchange interactions have been studied as well, ${ }^{7-17}$ interpolating from the triangular lattice to both the square-lattice and the decoupled-chain limits, and both VBS and spin-liquid phases have been proposed to occur.

Experimentally, triangular-lattice Heisenberg models are realized in a variety of materials, such as the inorganic Mott insulators $\mathrm{Cs}_{2} \mathrm{CuCl}_{4}$ and $\mathrm{Cs}_{2} \mathrm{CuBr}_{4}$ (Refs. 18 and 19) and the organic compounds $\kappa-(\mathrm{ET})_{2} \mathrm{Cu}_{2}(\mathrm{CN})_{3}$ (Ref. 20) and $X\left[\mathrm{Pd}(\mathrm{dmit})_{2}\right]_{2}$ (Refs. 21-25, with $X=\mathrm{EtMe}_{3} \mathrm{Sb}$ or $\mathrm{EtMe}_{3} \mathrm{P}$ ), all showing some degree of spatial anisotropy. In the latter two, the crystal lattice is rather soft: pressure can be used to induce an insulator-to-metal transition, with superconductivity appearing at low temperature. Spin-lattice interactions are also relevant in the insulator, as they potentially relieve frustration via dimerization. In fact, such a dimerized VBS phase has been reported for $\mathrm{EtMe}_{3} \mathrm{P}\left[\mathrm{Pd}(\mathrm{dmit})_{2}\right]_{2}$, likely stabilized by magnetoelastic coupling.
PACS number(s): 75.10.Jm, 75.10.Kt, 75.30.Kz, 75.50.Ee

In this paper, we study the columnar dimer phase of a generic triangular-lattice antiferromagnet (AF). ${ }^{26}$ In the absence of detailed knowledge about microscopics, we focus on a simple, yet very rich, model [Fig. 1(a)] with anisotropic nearest-neighbor couplings and explicit dimerization, the latter possibly arising from magnetoelastic effects. A determination of the full phase diagram of this model (see Fig. 2 for a sketch) is a hard task. Here we stick to the more modest goal of characterizing the dimer phase and its triplon excitations and locating the line of quantum phase transitions (QPTs) to a magnetically ordered state, where the triplon gap closes. To this end, we employ the bond-operator technique introduced by Sachdev and Bhatt. ${ }^{27}$ It turns out to be important to go beyond the linearized (i.e., noninteracting) boson problem: We discuss various approximation schemes, also providing a guideline as to which nonlinear effects are important in the presence of frustration.

Among our results is the existence of a commensurateincommensurate transition (CIT) in the excitation spectrum, i.e., the minimum of the triplon dispersion is locked at wave vector $(0,0)$ (in the coordinates of the dimerized lattice, Fig. 1) in some region of parameter space, while it moves to incommensurate momenta elsewhere. If the triplon gap closes at the CIT line, the excitations become anomalously soft, leading to a quantum Lifshitz point. ${ }^{28,29} \mathrm{We}$ briefly discuss the unusual quantum critical regime of such a putative magnetic QPT, but we also speculate about an intervening nontrivial paramagnetic phase. Finally, we discuss two-particle (as opposed to three-particle) decay of magnetic excitations, ${ }^{30,31}$ which is generically possible in low-symmetry dimerized magnets.

\section{A. Model}

We consider a triangular-lattice Heisenberg model,

$$
\mathcal{H}=\sum_{\langle i, j\rangle} J_{i j} \mathbf{S}_{i} \cdot \mathbf{S}_{j},
$$


(a)

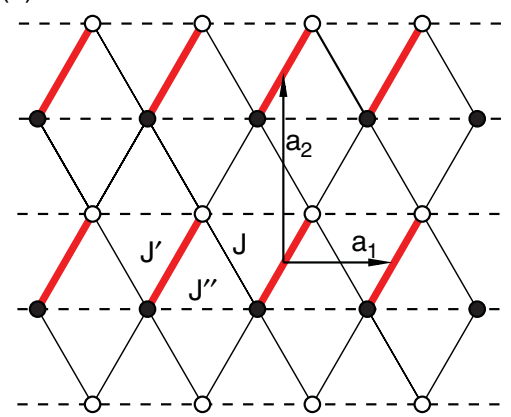

(b)

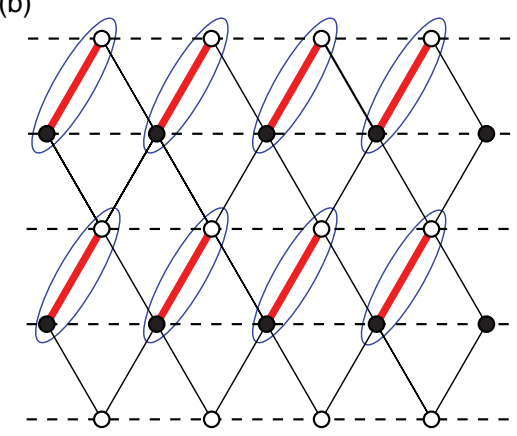

(c)

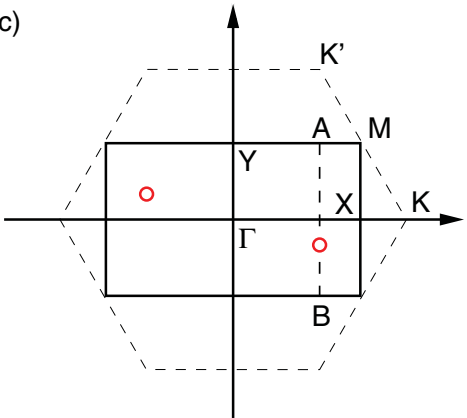

FIG. 1. (Color online) (a) Lattice structure of the model (1), with exchange couplings $J, J^{\prime}$, and $J^{\prime \prime}$ shown as black (thin), red (thick), and dashed black lines, respectively. Solid (open) circles represent spin $\mathbf{S}^{1}\left(\mathbf{S}^{2}\right)$ of each dimer. $\mathbf{a}_{1}$ and $\mathbf{a}_{2}$ are the primitive vectors of the dimerized lattice. (b) Dimerized ground state in the limit of large $J^{\prime}$. Here, ellipses represents a singlet. (c) Brillouin zone of the dimerized lattice (solid), together with the (hexagonal) Brillouin zone of the original triangular lattice (dashed). The open circles indicate the minimum position of $\omega_{\mathbf{q}}$ [Eq. (17)] for parameters $J^{\prime}=1.5$ and $J^{\prime \prime}=1$. Here $\mathbf{K}=(4 \pi / 3 a, 0), \mathbf{K}^{\prime}=(2 \pi / 3 a, 2 \pi / \sqrt{3} a), \mathbf{X}=(\pi / a, 0), \mathbf{Y}=(0, \pi / \sqrt{3} a)$, and $\mathbf{M}=(\pi / a, \pi / \sqrt{3} a)$, with $a$ being the lattice spacing of the triangular lattice.

where $\mathbf{S}_{i}$ represents a spin-1/2 at site $i$, and the nearestneighbor exchange couplings $J_{i j}$ equal $J, J^{\prime}$, and $J^{\prime \prime}$ according to the pattern shown in Fig. 1(a). ${ }^{26}$ Hereafter, we employ $J=1$.

\section{B. Phase diagram}

To appreciate the richness of the model (1), it is useful to discuss a few limiting cases, with their properties summarized in the schematic phase diagram in Fig. 2.

(i) Line $J^{\prime \prime}=1$. On this line, for $J^{\prime}=1$, we recover the isotropic triangular-lattice AF Heisenberg model. Its ground state has noncollinear $120^{\circ}$ Néel order, with commensurate ordering wave vectors $\mathbf{K}=(4 \pi / 3 a, 0)$ and $\mathbf{K}^{\prime}=$ $(2 \pi / 3 a, 2 \pi / \sqrt{3} a) .5$ Noncollinear order can be expected to extend to small values of $J^{\prime}$. On the other hand, the limit $J^{\prime} \gg 1$ corresponds to a paramagnet of weakly coupled dimers, ${ }^{32}$ such that spins that are pairwise coupled by $J^{\prime}$ combine into a columnar arrangement of singlets, as illustrated in Fig. 1(b).

(ii) Line $J^{\prime \prime}=0$. Model (1) is now topologically equivalent to the staggered dimerized Heisenberg model on a square lattice, recently discussed in Refs. 33 and 34. According to quantum Monte Carlo (QMC) calculations, a order-disorder QPT takes place at $J_{c}^{\prime}=2.5196,{ }^{33}$ with collinear Néel order setting in for $J^{\prime}<J_{c}^{\prime}$. The line $J^{\prime \prime}=0$ also includes the square and honeycomb lattice $\mathrm{AF}$ at $J^{\prime}=1$ and 0 , respectively.

(iii) Line $J^{\prime}=1$. Here, Eq. (1) represents the spatially anisotropic triangular-lattice AF addressed in Refs. 7-17. In particular, coupled-cluster calculations ${ }^{10}$ indicate that the system displays collinear Néel order for $J^{\prime \prime}<0.8$ and noncollinear spiral order with an incommensurate ordering wave vector (except at $J^{\prime \prime}=1$ ) for $0.8<J^{\prime \prime}<1.8$. For $J^{\prime \prime} \gg 1$, the system consists of weakly coupled chains, and a collinear $\mathrm{AF}$ state $^{10}$ has been argued to arise as a result of order-from-disorder physics. ${ }^{11,12}$ However, we note that both noncollinear ${ }^{13}$ and disordered (i.e., spin-liquid) ground states $^{14,15}$ have also been proposed in this regime. For $0.7<$ $J^{\prime \prime}<0.9$, the physics is under debate as well: series-expansion studies favored a spontaneously dimerized VBS in this region, ${ }^{9}$ which was not found in the coupled-cluster study. ${ }^{10}$

In total, the phase diagram of the model (1), Fig. 2, displays a gapped paramagnetic phase, both collinear and noncollinear long-range order (LRO), as well as putative nontrivial spinliquid regimes that may or may not be adiabatically connected to the one-dimensional limit $J^{\prime \prime} \rightarrow \infty$.

In this paper, our focus is on the properties of the dimerized paramagnet, which is adiabatically connected to the limit of large $J^{\prime}$. As stated above, in a real system, such as $\mathrm{EtMe}_{3} \mathrm{P}\left[\mathrm{Pd}(\mathrm{dmit})_{2}\right]_{2}$, the dimerization may arise spontaneously due to longer-range or ring-exchange couplings, and will then be stabilized by magnetoelastic effects. Leaving a detailed study of the latter for the future, we choose to work with explicit dimerization as in Fig. $1 .{ }^{26}$ Most of our calculations are restricted to the parameter range $J^{\prime}>J^{\prime \prime}$ and $0 \leqslant J^{\prime \prime} \leqslant 1.5$. A quantitative phase diagram obtained from various bond-operator approximations is shown in Fig. 3 and will be discussed in detail below.

\section{Outline}

The remainder of our paper is organized as follows: In Sec. II, we briefly summarize the bond-operator formalism that is employed to describe the paramagnetic phase of the model (1) and derive an effective Hamiltonian of interacting triplet excitations (triplons). In Sec. III, we analyze the noninteracting (i.e., harmonic) part of this Hamiltonian. We determine the magnitude and momentum-space location of the minimum energy gap of the triplons. Corrections arising from interactions - both cubic and quartic_-among the triplons are analyzed in some detail in Sec. IV. For all levels of approximation, the closing of the triplon gap allows us to construct the phase boundary between the singlet and long-range-ordered phases, shown in Fig. 3. In Sec. V, we discuss various aspects of our results, such as the two-particle decay of triplons due to cubic interactions, the commensurate-incommensurate transition, and the associated quantum Lifshitz point. We also comment on some implications for the organic Mott insulator $\mathrm{EtMe}_{3} \mathrm{P}\left[\mathrm{Pd}(\mathrm{dmit})_{2}\right]_{2}$. Concluding remarks close the paper. A 


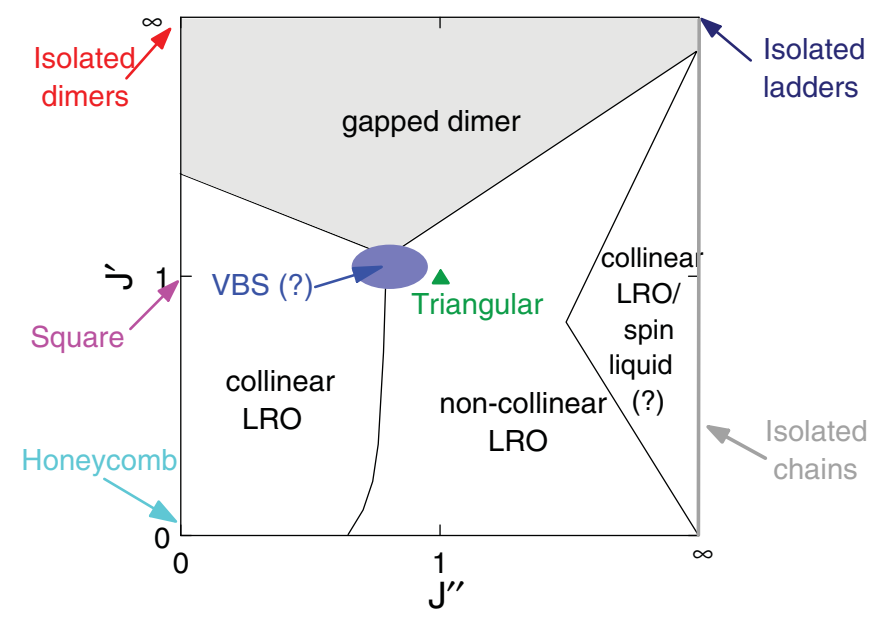

FIG. 2. (Color online) Schematic phase diagram of the Heisenberg model (1) as a function of the couplings $J^{\prime}$ and $J^{\prime \prime}$, keeping $J=1$. For large $J^{\prime}$, a gapped dimer phase is realized (shaded). Various other limits are indicated in the figure; see text for details. The multicritical point, where the collinear LRO, noncollinear LRO, and gapped dimer phases meet, is the quantum Lifshitz point. [The classical limit of (1) is discussed in Appendix A.]

summary of the classical phase diagram of (1) as well as details of the calculations are relegated to the Appendixes.

\section{BOND OPERATORS AND TRIPLON EXCITATIONS}

For $J^{\prime}>J, J^{\prime \prime}$, it is useful to view the spin pairs coupled by $J^{\prime}$ as building blocks of the model. The four states per such dimer can be efficiently represented using bond operators, ${ }^{27}$ which naturally lead to a description of the elementary excitations of the paramagnetic phase in terms of bosonic spin-1 modes, dubbed "triplons."

\section{A. Bond-operator representation}

To introduce bond operators, the triangular lattice of spins is reinterpreted as a rectangular lattice of dimers, Fig. 1(b), with sites $i$. For each dimer, one introduces bosonic operators $\left\{s_{i}^{\dagger}, t_{i \alpha}^{\dagger}\right\}(\alpha=x, y, z)$, which create the dimer states out of a fictitious vacuum. Explicitly (and omitting the site index $i$ ), $|s\rangle=s^{\dagger}|0\rangle$ and $|\alpha\rangle=t_{\alpha}^{\dagger}|0\rangle$, where $|s\rangle=(|\uparrow \downarrow\rangle-|\downarrow \uparrow\rangle) / \sqrt{2}$, $|x\rangle=(-|\uparrow \uparrow\rangle+|\downarrow \downarrow\rangle) / \sqrt{2},|y\rangle=i(|\uparrow \uparrow\rangle+|\downarrow \downarrow\rangle) / \sqrt{2}$, and $|z\rangle=(|\uparrow \downarrow\rangle+|\downarrow \uparrow\rangle) / \sqrt{2}$. The Hilbert-space dimension is conserved by imposing the constraint

$$
s_{i}^{\dagger} s_{i}+\sum_{\alpha} t_{i \alpha}^{\dagger} t_{i \alpha}=1
$$

on every site $i$. The original spin operators $\mathbf{S}^{1}$ and $\mathbf{S}^{2}$ of each dimer are given by

$$
S_{\alpha}^{1,2}= \pm \frac{1}{2}\left(s^{\dagger} t_{\alpha}+t_{\alpha}^{\dagger} s \mp i \epsilon_{\alpha \beta \gamma} t_{\beta}^{\dagger} t_{\gamma}\right)
$$

where $\epsilon_{\alpha \beta \gamma}$ is the antisymmetric tensor with $\epsilon_{x y z}=1$ and summation convention over repeated indices is implied.

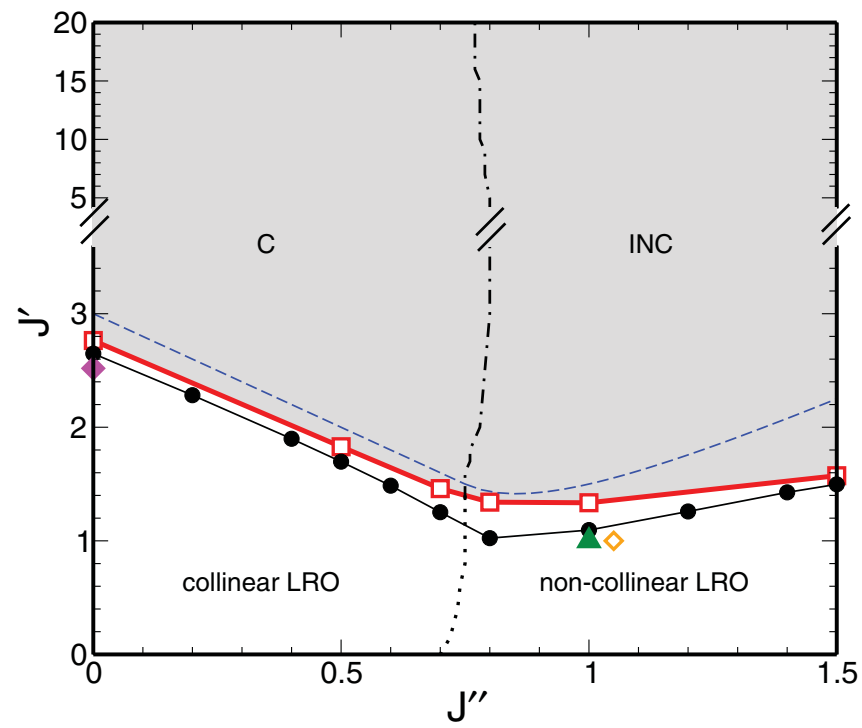

FIG. 3. (Color online) Quantitative phase diagram for the Heisenberg model (1), obtained using the bond-operator formalism. The dimer phase boundary ( $\square$ ) has been obtained from the HF-cubic approximation described in the paper; for comparison, we also show the results of the harmonic mean-field ${ }^{27}(\bullet)$ and harmonic spin-wave approximations (dashed blue). ${ }^{37}$ The dashed-dotted line separates the commensurate $(\mathrm{C})$ and incommensurate (INC) regions of the dimer phase at large $J^{\prime}$. It continues into the dotted line, which separates the phases with collinear and noncollinear LRO at small $J^{\prime}$-note that this line is an estimate based on series-expansion ${ }^{9}$ and classical-limit results (Appendix A). Also shown are the isotropic point $J^{\prime}=J^{\prime \prime}=1$ $(\Delta)$ and the point $J^{\prime}=1, J^{\prime \prime}=1.05(\nabla)$ for $\mathrm{EtMe}_{3} \mathrm{P}\left[\mathrm{Pd}(\mathrm{dmit})_{2}\right]_{2} \cdot{ }^{22}$ (Here, longer-range or multiple-spin interactions can also be expected to be relevant.) Finally, on the $J^{\prime \prime}=0$ axis we have indicated the QMC value of $J_{c}^{\prime}(\diamond)$ for the square-lattice staggered dimerized Heisenberg model. ${ }^{33}$

\section{B. Effective theory for triplet excitations}

Expressing the Hamiltonian (1) in terms of the dimer spins $\left(\mathbf{S}_{i}^{1}\right.$ and $\left.\mathbf{S}_{i}^{2}\right)$ yields

$$
\begin{aligned}
\mathcal{H}= & \sum_{i}\left[J^{\prime} \mathbf{S}_{i}^{1} \cdot \mathbf{S}_{i}^{2}+J^{\prime \prime}\left(\mathbf{S}_{i}^{1} \cdot \mathbf{S}_{i+1}^{1}+\mathbf{S}_{i}^{2} \cdot \mathbf{S}_{i+1}^{2}\right)\right] \\
& +J \sum_{i, n} \mathbf{S}_{i}^{2} \cdot \mathbf{S}_{i+n}^{1} .
\end{aligned}
$$

Here $n=1,2,3$ corresponds to the nearest-neighbor vectors

$$
\boldsymbol{\tau}_{1}=a \hat{x}, \quad \boldsymbol{\tau}_{2}=\sqrt{3} a \hat{y}, \quad \boldsymbol{\tau}_{3}=a(\hat{x}+\sqrt{3} \hat{y}),
$$

with $a$ being the lattice spacing of the original triangular lattice (in the following, $a=1$ ). Substituting Eq. (3) into (4) yields a Hamiltonian of the form

$$
\mathcal{H}=\mathcal{H}_{0}+\mathcal{H}_{2}+\mathcal{H}_{3}+\mathcal{H}_{4},
$$

where $\mathcal{H}_{n}$ contains $n$ triplet operators (see Appendix B for details).

In the paramagnetic phase realized for large $J^{\prime}$, the singlet can be viewed as "condensed," 27 formally $s_{i}^{\dagger}, s_{i} \rightarrow \sqrt{N_{0}}$ in Eq. (6). As a consequence, one ends up with an effective Hamiltonian solely in terms of the (bosonic) triplet operators $t_{i \alpha}^{\dagger}$. In the spirit of mean-field theory, the constraint (2) is 
implemented on average via a Lagrange multiplier $\mu$. Both $\mu$ and $N_{0}$ will be self-consistently determined. One expects that $N_{0} \approx 1$ in the limit $J^{\prime} \gg J, J^{\prime \prime}$. (Other bond-operator schemes will be discussed in Sec. II C below.)

After performing a Fourier transform, i.e., $t_{i \alpha}^{\dagger}=$ $N^{\prime-1 / 2} \sum_{\mathbf{k}} \exp \left(-i \mathbf{k} \cdot \mathbf{R}_{i}\right) t_{\mathbf{k} \alpha}^{\dagger}$, the terms of the Hamiltonian (6) read

$$
\begin{aligned}
& E_{0}=-3 J^{\prime} N / 8-\mu N\left(N_{0}-1\right) / 2, \\
& \mathcal{H}_{2}=\sum_{\mathbf{k}}\left[A_{\mathbf{k}} t_{\mathbf{k} \alpha}^{\dagger} t_{\mathbf{k} \alpha}+\frac{1}{2} B_{\mathbf{k}}\left(t_{\mathbf{k} \alpha}^{\dagger} t_{-\mathbf{k} \alpha}^{\dagger}+\text { H.c. }\right)\right], \\
& \mathcal{H}_{3}=\frac{1}{2 \sqrt{N^{\prime}}} \epsilon_{\alpha \beta \lambda} \sum_{\mathbf{p}, \mathbf{k}} \xi_{\mathbf{k}-\mathbf{p}} t_{\mathbf{k}-\mathbf{p} \alpha}^{\dagger} t_{\mathbf{p} \beta}^{\dagger} t_{\mathbf{k} \lambda}+\text { H.c. } \\
& \mathcal{H}_{4}=\frac{1}{2 N^{\prime}} \epsilon_{\alpha \beta \lambda} \epsilon_{\alpha \mu \nu} \sum_{\mathbf{q}, \mathbf{p}, \mathbf{k}} \gamma_{\mathbf{k}} t_{\mathbf{p}+\mathbf{k} \beta}^{\dagger} t_{\mathbf{q}-\mathbf{k} \mu}^{\dagger} t_{\mathbf{q} \nu} t_{\mathbf{p} \lambda} .
\end{aligned}
$$

Here $N^{\prime}=N / 2$, with $N$ being the number of sites of the original triangular lattice, and the momentum sum runs over the dimerized (rectangular) Brillouin zone. The coefficients $A_{\mathbf{k}}, B_{\mathbf{k}}, \xi_{\mathbf{k}}$, and $\gamma_{\mathbf{k}}$ are given by

$A_{\mathrm{k}}=\frac{J^{\prime}}{4}-\mu+B_{\mathrm{k}}$

$B_{\mathbf{k}}=\frac{1}{2} N_{0}\left[\left(2 J^{\prime \prime}-1\right) \cos k_{x}-\cos \left(\sqrt{3} k_{y}\right)-\cos \left(k_{x}+\sqrt{3} k_{y}\right)\right]$,

$\xi_{\mathbf{k}}=-\sqrt{N_{0}}\left[\sin k_{x}+\sin \left(\sqrt{3} k_{y}\right)+\sin \left(k_{x}+\sqrt{3} k_{y}\right)\right]$,

$\gamma_{\mathbf{k}}=-\frac{1}{2}\left[\left(2 J^{\prime \prime}+1\right) \cos k_{x}+\cos \left(\sqrt{3} k_{y}\right)+\cos \left(k_{x}+\sqrt{3} k_{y}\right)\right]$.

A few remarks here about the general structure of the effective Hamiltonian (6) are in order: The bond-operator approach has some parallels to the Holstein-Primakoff approach to ordered magnets, with the difference that one considers fluctuations above a quantum paramagnetic state instead of a Néel state. A cubic triplet term (8) is present in many lowsymmetry situations, including the model considered here and also the square-lattice staggered dimerized Heisenberg model of Refs. 33 and 34. This is to be contrasted with spin waves, where a cubic interaction term-describing two-particle decay of transverse magnons-is only present for noncollinear spin structures while it vanishes for collinear ones (see Ref. 35 for a discussion).

\section{Alternative bond-operator schemes}

The procedure discussed in the preceding section, where the constraint (2) is included into the description via a Lagrange multiplier and $N_{0}$ is self-consistently determined, is the one originally proposed by Sachdev and Bhatt. ${ }^{27}$ However, alternative methods to deal with the constraint (2) can be found in the literature. Let us briefly summarize two of them.

Chubukov and Morr $^{36}$ resolve the constraint via $s=\left(1-\lambda t_{\alpha}^{\dagger} t_{\alpha}\right)^{1 / 2}$, where $\lambda$ is an artificial control parameter, with $\lambda=1$ corresponding to the physical case. The square root can now be expanded to obtain a Taylor series in the control parameter $\lambda-$ a procedure similar to the $1 / S$ expansion in conventional spin-wave theory. This generates a Hamiltonian with triplon terms up to arbitrary order, which can be analyzed order by order in $\lambda$. Finally, one arrives at physical results by taking the limit $\lambda \rightarrow 1$.

Kotov et al. ${ }^{37}$ instead implement the hard-core constraint explicitly in the following way. First, the $t$ operators are reinterpreted as creation operators of triplons on top of a singlet background. This is formally equivalent to setting $s_{i}^{\dagger}=s_{i}=1$. The resulting Hamiltonian contains terms up to quartic order; its harmonic piece, $\mathcal{H}_{2}$, is analogous to linear spin-wave theory. The constraint is converted into the inequality $\sum_{\alpha} t_{i \alpha}^{\dagger} t_{i \alpha} \leqslant 1$, which can be imposed using an infinite on-site triplet repulsion term

$$
\mathcal{H}_{U}=\frac{1}{2 N^{\prime}} U \sum_{i, \alpha, \beta} t_{i, \alpha}^{\dagger} t_{i, \beta}^{\dagger} t_{i, \beta} t_{i, \alpha}, \quad U \rightarrow \infty .
$$

$\mathcal{H}_{U}$, which provides the main contribution to the renormalization of the noninteracting triplet energy, is treated using a self-consistent ladder summation of diagrams (Brueckner approximation).

Both schemes were applied to the nonfrustrated squarelattice bilayer Heisenberg model, with the Brueckner approach $^{37}$ giving very accurate results, e.g., for the location of the phase boundary. Results of similar quality were obtained for other models with collinear spin correlations. ${ }^{38}$ In contrast, we have found that the Brueckner approach is less well suited for the present triangular-lattice model: Following Ref. 37, we have implemented the Brueckner approximation for the hard-core triplon repulsion and included a non-self-consistent treatment of the cubic term to second order. In the resulting phase diagram, the stability of the paramagnetic phase is clearly overestimated, i.e., we find the gapped paramagnet to be stable even at the isotropic point $J^{\prime}=J^{\prime \prime}=1$, with the critical $J_{c}^{\prime} \approx 0.75$ at $J^{\prime \prime}=1$. We suspect that the combined effect of the hard-core and cubic terms in the presence of noncollinear correlations requires a more accurate approximation, but we have not explored this route further. Therefore, we resort to the mean-field-based approach of Ref. 27, the results of which are presented in the body of the paper.

\section{HARMONIC APPROXIMATION}

The lowest-order approximation to the triplon dynamics consists in keeping the quadratic term $\mathcal{H}_{2}$ of the Hamiltonian only, which describes the physics of noninteracting bosons. $\mathcal{H}_{2}$ can be diagonalized with the help of the Bogoliubov transformation

$$
t_{\mathbf{k} \alpha}^{\dagger}=u_{\mathbf{k}} b_{\mathbf{k} \alpha}^{\dagger}-v_{\mathbf{k}} b_{-\mathbf{k} \alpha} .
$$

One finds

$$
\mathcal{H}=\bar{E}_{0}+\sum_{\mathbf{k} \alpha} \omega_{\mathbf{k}} b_{\mathbf{k} \alpha}^{\dagger} b_{\mathbf{k} \alpha}
$$

where

$$
\bar{E}_{0}=-\frac{3}{8} J^{\prime} N N_{0}-\frac{1}{2} \mu N\left(N_{0}-1\right)+\frac{3}{2} \sum_{\mathbf{k}}\left(\omega_{\mathbf{k}}-A_{\mathbf{k}}\right)
$$


is the ground state energy,

$$
\omega_{\mathbf{k}}=\sqrt{A_{\mathbf{k}}^{2}-B_{\mathbf{k}}^{2}}
$$

is the energy of the triplet excitations, and the Bogoliubov coefficients in Eq. (14) obey

$$
u_{\mathbf{k}}^{2}, v_{\mathbf{k}}^{2}= \pm 1 / 2+A_{\mathbf{k}} / 2 \omega_{\mathbf{k}} \quad \text { and } \quad u_{\mathbf{k}} v_{\mathbf{k}}=B_{\mathbf{k}} / 2 \omega_{\mathbf{k}} .
$$

From the saddle-point conditions $\partial E_{0} / \partial N_{0}=0$ and $\partial E_{0} / \partial \mu=0$, self-consistent equations for $\mu$ and $N_{0}$ follow (see Appendix C for details),

$$
\begin{gathered}
\mu=-\frac{3 J^{\prime}}{4}+\frac{3}{N_{0}} \sum_{\mathbf{k}} B_{\mathbf{k}} v_{\mathbf{k}}\left(v_{\mathbf{k}}-u_{\mathbf{k}}\right), \\
N_{0}=1-\frac{3}{N^{\prime}} \sum_{\mathbf{k}} v_{\mathbf{k}}^{2}=1-\frac{1}{N^{\prime}} \sum_{\mathbf{k} \alpha}\left\langle t_{\mathbf{k} \alpha}^{\dagger} t_{\mathbf{k} \alpha}\right\rangle .
\end{gathered}
$$

Once $\mu$ and $N_{0}$ are known, the triplon energy (17) is completely determined.

In Fig. 4, we show the triplon dispersion relation for two sets of model parameters inside the disordered phase. For the case $J^{\prime}=3$ and $J^{\prime \prime}=0$, the minimum gap is located at $\Gamma$, the center of the dimerized Brillouin zone, while for $J^{\prime}=1.5$ and $J^{\prime \prime}=1.0$, the minimum gap is at incommensurate momenta, $\mathbf{Q}_{0}= \pm(2 \pi / 3,-\pi / 3 \sqrt{3})$. Indeed, within the harmonic approximation, the momentum $\mathbf{Q}_{0}$ associated with the triplon gap can be analytically calculated. From the solution of $\nabla_{\mathbf{q}} \omega_{\mathbf{q}}=\nabla_{\mathbf{q}} B_{\mathbf{q}}=0$, one finds that it depends only on the coupling $J^{\prime \prime}$, namely

$$
\mathbf{Q}_{0}= \begin{cases}(0,0), & 0 \leqslant J^{\prime \prime} \leqslant 0.75 \\ \pm\left(q_{\mathrm{xm}}, q_{\mathrm{ym}}\right), & J^{\prime \prime}>0.75\end{cases}
$$

with

$$
\begin{aligned}
& q_{\mathrm{xm}}=2 \arccos \left(\frac{1}{4 J^{\prime \prime}-2}\right), \\
& q_{\mathrm{ym}}=-\frac{1}{\sqrt{3}} \arccos \left(\frac{1}{4 J^{\prime \prime}-2}\right) .
\end{aligned}
$$

Note that $\mathbf{Q}_{0}$ continuously moves from a commensurate, $(0,0)$, to an incommensurate point, $\pm\left(q_{\mathrm{xm}}, q_{\mathrm{ym}}\right)$, as $J^{\prime \prime}$ increases, defining a commensurate-incommensurate transition (CIT) in the excitation spectrum at $J_{\mathrm{CIT}}^{\prime \prime}=0.75$; see Fig. 5. At the present harmonic level, $\mathbf{Q}_{0}$ only depends on $J^{\prime \prime}$, but this does (a)

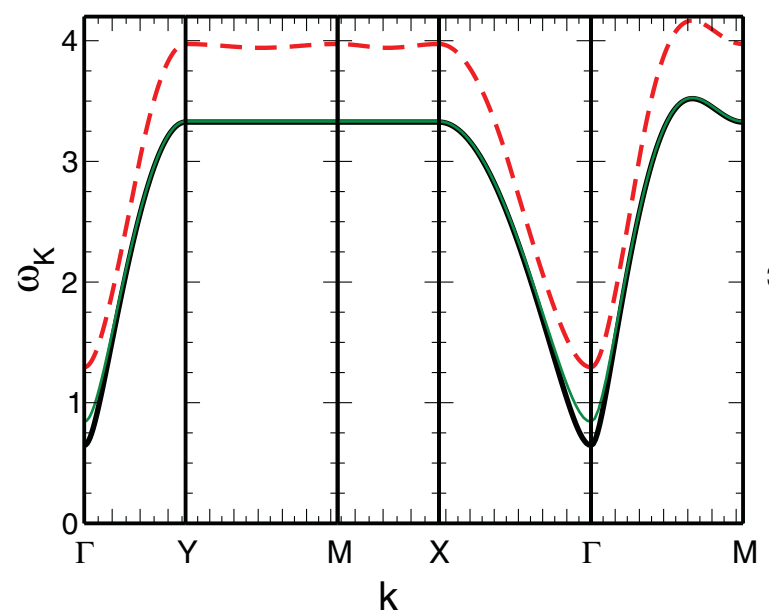

(c)

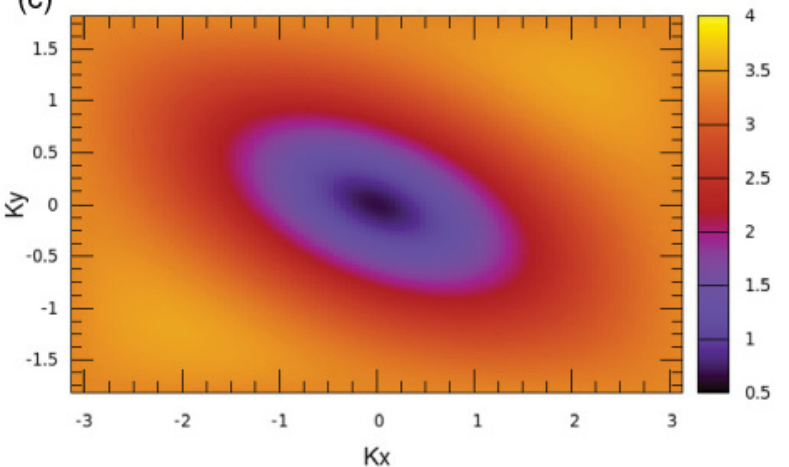

(b)
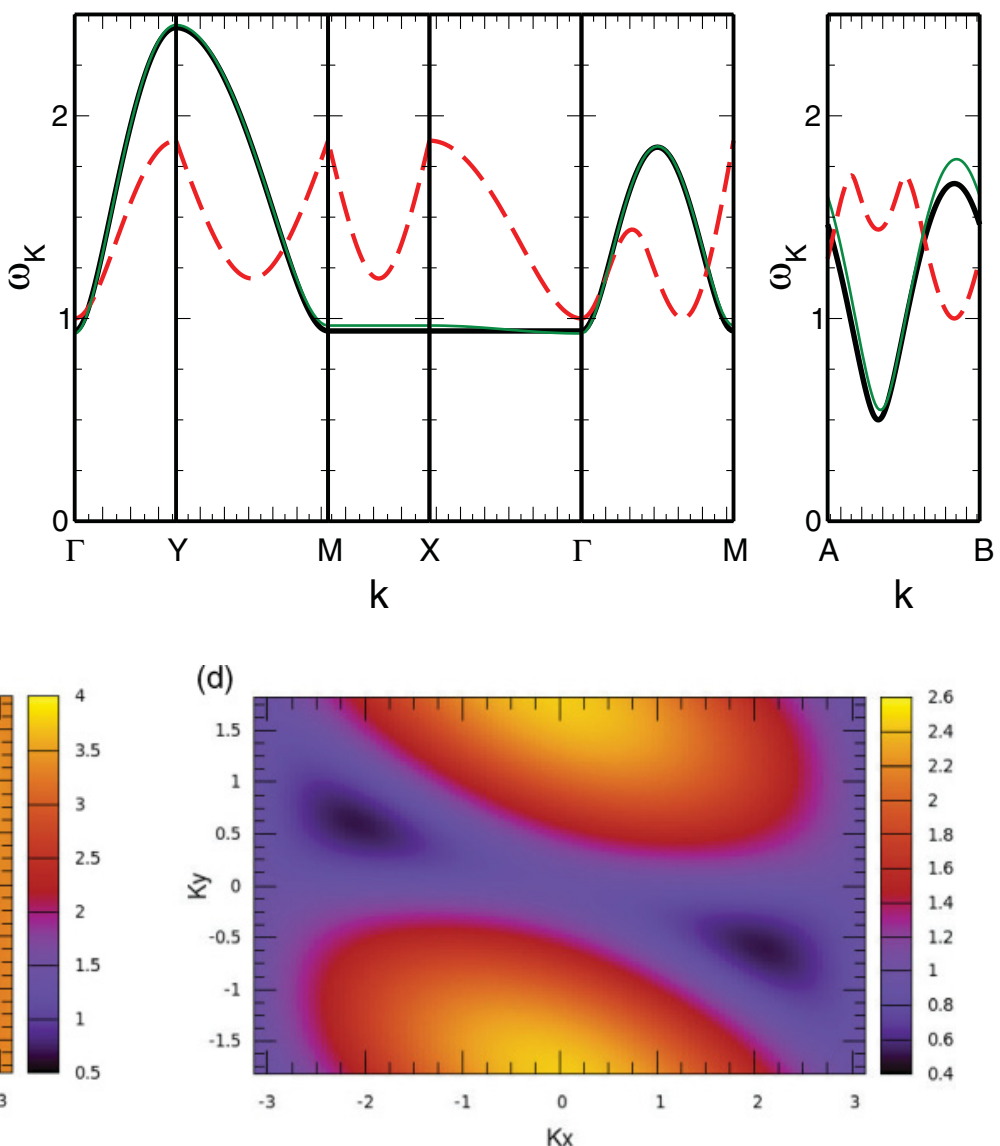

FIG. 4. (Color online) Upper row: Triplon dispersion relation at the harmonic level (solid black) along paths in the dimerized Brillouin zone [Fig. 1(c)], for (a) $J^{\prime}=3.0, J^{\prime \prime}=0$ and (b) $J^{\prime}=1.5, J^{\prime \prime}=1$. For comparison, the dispersion obtained within the HF approximation for the quartic term (thin green line, see Sec. IV A) is also shown, indicating that the corrections from the quartic term are minor. Finally, the dashed red line represents the bottom of the two-particle continuum at the harmonic level. Lower row: Contour plot of the triplon energy at the harmonic level, for (c) $J^{\prime}=3.0, J^{\prime \prime}=0$ and (d) $J^{\prime}=1.5, J^{\prime \prime}=1$. 
(a)

$$
J^{\prime}=1.7
$$
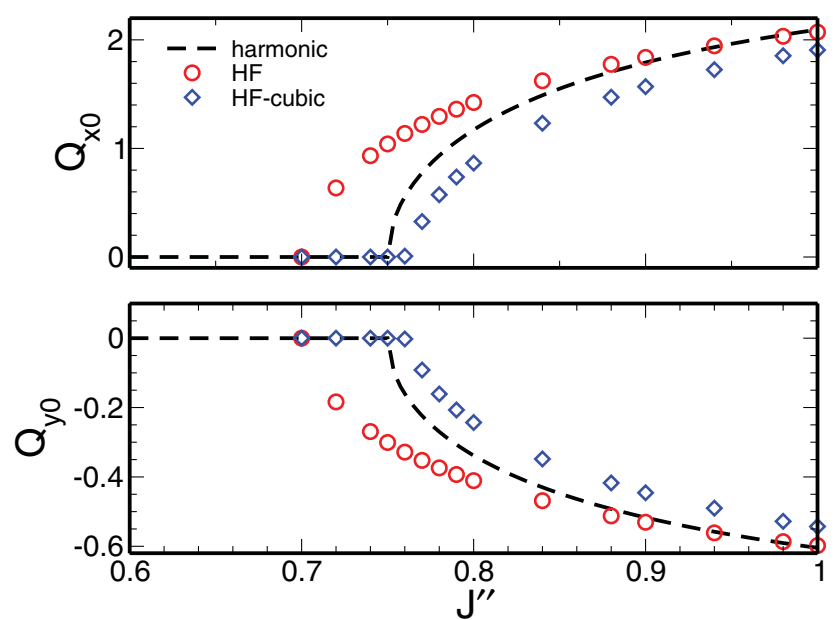

(b)
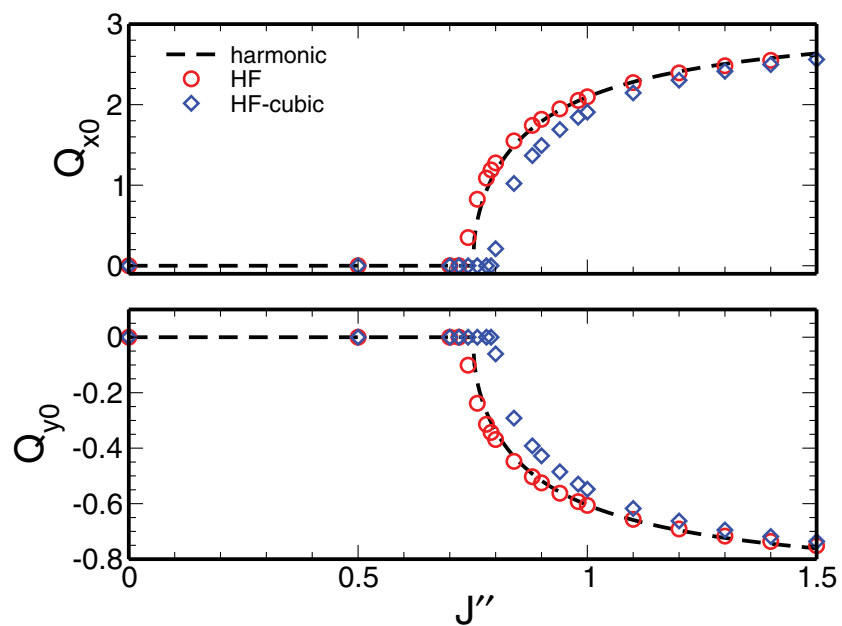

FIG. 5. (Color online) $x$ and $y$ components of the momentum $\mathbf{Q}_{0}$ (see text for definition) as a function of $J^{\prime \prime}$ at the harmonic (dashed), HF $(\bigcirc)$, and HF-cubic $(\diamond)$ approximations for the lines $J^{\prime}=1.7$ (a) and 3.0 (b). Nonzero values of $\mathbf{Q}_{0}$ correspond to incommensurate correlations.

not hold once corrections are included; see Sec. IV. Further aspects of the CIT will be discussed in Sec. V B below.

Parenthetically, we note that—at the harmonic level—one recovers the spin-wave-type approximation of Kotov et al. ${ }^{37}$ (see Sec. IIC) if, instead of calculating $\mu$ and $N_{0}$ selfconsistently, one sets $\mu=-3 J^{\prime} / 4$ and $N_{0}=1$.

\section{TRIPLON INTERACTIONS}

Both the cubic and quartic interaction terms, $\mathcal{H}_{3}(8)$ and $\mathcal{H}_{4}(9)$, renormalize the triplon energy and, consequently, shift the phase boundary of the paramagnetic phase. Moreover, the cubic term also induces two-particle decay of triplons, as discussed in Refs. 30 and 31. In order to include triplon-triplon interactions into our description, we treat the effect of $\mathcal{H}_{4}$ at the mean-field level (Sec. IV A) and the one of $\mathcal{H}_{3}$ perturbatively (Sec. IV B).

\section{A. Hartree-Fock approximation}

We treat the quartic triplon interaction within the selfconsistent Hartree-Fock (HF) approximation. It is straightforward to show that Eq. (9) assumes the form (for details, see Appendix A of Ref. 35)

$$
\mathcal{H}_{4}^{\mathrm{HF}}=E_{0}^{\mathrm{HF}}+\sum_{\mathbf{k}}\left[A_{\mathbf{k}}^{\mathrm{HF}} t_{\mathbf{k} \alpha}^{\dagger} t_{\mathbf{k} \alpha}+\frac{1}{2} B_{\mathbf{k}}^{\mathrm{HF}}\left(t_{\mathbf{k} \alpha}^{\dagger} t_{-\mathbf{k} \alpha}^{\dagger}+\text { H.c. }\right)\right] .
$$

Here

$$
\begin{aligned}
& E_{0}^{\mathrm{HF}}=\frac{3}{N^{\prime}} \sum_{\mathbf{k} \mathbf{q}} \gamma_{\mathbf{k}}\left(\bar{v}_{\mathbf{k}+\mathbf{q}}^{2} \bar{v}_{\mathbf{q}}^{2}-\bar{v}_{\mathbf{k}+\mathbf{q}} \bar{u}_{\mathbf{k}+\mathbf{q}} \bar{v}_{\mathbf{q}} \bar{u}_{\mathbf{q}}\right), \\
& A_{\mathbf{k}}^{\mathrm{HF}}=-\frac{2}{N^{\prime}} \sum_{\mathbf{q}} \gamma_{\mathbf{q}-\mathbf{k}} \bar{v}_{\mathbf{q}}^{2}=\sum_{n=1}^{3} A_{n}^{\mathrm{HF}} \cos \left(\mathbf{k} \cdot \boldsymbol{\tau}_{n}\right), \\
& B_{\mathbf{k}}^{\mathrm{HF}}=-\frac{2}{N^{\prime}} \sum_{\mathbf{q}} \gamma_{\mathbf{q}-\mathbf{k}} \bar{u}_{\mathbf{q}} \bar{v}_{\mathbf{q}}=\sum_{n=1}^{3} B_{n}^{\mathrm{HF}} \cos \left(\mathbf{k} \cdot \boldsymbol{\tau}_{n}\right),
\end{aligned}
$$

with $\gamma_{\mathbf{k}}$ being the bare quartic vertex (13), $\bar{u}_{\mathbf{q}}$ and $\bar{v}_{\mathbf{q}}$ the Bogoliubov coefficients (see definition below), $\boldsymbol{\tau}_{n}$ the nearestneighbor vectors (5), and the coefficients $A_{n}^{\mathrm{HF}}$ read

$$
\begin{aligned}
& A_{1}^{\mathrm{HF}}=\left(J^{\prime \prime}+2\right) \frac{1}{N^{\prime}} \sum_{\mathbf{k}} \cos \left(k_{x}\right) \bar{u}_{\mathbf{k}}^{2}, \\
& A_{2}^{\mathrm{HF}}=\frac{1}{N^{\prime}} \sum_{\mathbf{k}} \cos \left(\sqrt{3} k_{y}\right) \bar{u}_{\mathbf{k}}^{2}, \\
& A_{3}^{\mathrm{HF}}=\frac{1}{N^{\prime}} \sum_{\mathbf{k}} \cos \left(k_{x}+\sqrt{3} k_{y}\right) \bar{u}_{\mathbf{k}}^{2} .
\end{aligned}
$$

Similar expressions hold for $B_{n}^{\mathrm{HF}}$ but with $\bar{u}_{\mathbf{k}}^{2} \rightarrow \bar{u}_{\mathbf{k}} \bar{v}_{\mathbf{k}}$.

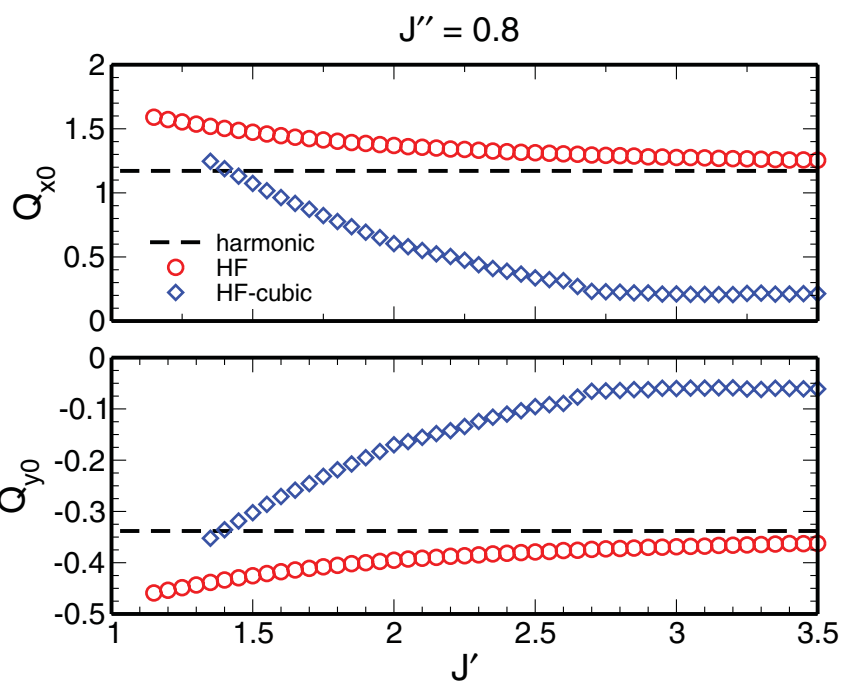

FIG. 6. (Color online) $x$ and $y$ components of the momentum $\mathbf{Q}_{0}$ (see text for definition) as a function of $J^{\prime}$ at the harmonic (dashed), HF $(\bigcirc)$, and HF-cubic $(\diamond)$ approximations for the line $J^{\prime \prime}=0.8$. In the strong-dimer limit of large $J^{\prime}$, the harmonic $\mathbf{Q}_{0}$ is approached for both the HF and HF-cubic results. 
The final Hamiltonian is now given by $\mathcal{H}=E_{0}+E_{0}^{\mathrm{HF}}+$ $\mathcal{H}_{2}+\mathcal{H}_{4}^{\mathrm{HF}}$. It is quadratic in triplet operators and can also be diagonalized by the Bogoliubov transformation (14), namely

$$
\mathcal{H}=\bar{E}_{0}^{\mathrm{HF}}+\sum_{\mathbf{k} \alpha} \bar{\omega}_{\mathbf{k}} b_{\mathbf{k} \alpha}^{\dagger} b_{\mathbf{k} \alpha} .
$$

In the above expression, the renormalized triplon energy $\bar{\omega}_{\mathbf{k}}$ is equal to Eq. (17) but now $A_{\mathbf{k}} \rightarrow \bar{A}_{\mathbf{k}}=A_{\mathbf{k}}+A_{\mathbf{k}}^{\mathrm{HF}}$ and $B_{\mathbf{k}} \rightarrow$ $\bar{B}_{\mathbf{k}}=B_{\mathbf{k}}+B_{\mathbf{k}}^{\mathrm{HF}}$. The ground-state energy $\bar{E}_{0}^{\mathrm{HF}}$ is similar to Eq. (16) apart from the replacements $\omega_{\mathbf{k}} \rightarrow \bar{\omega}_{\mathbf{k}}$ and $A_{\mathbf{k}} \rightarrow \bar{A}_{\mathbf{k}}$ and the inclusion of $E_{0}^{\mathrm{HF}}$. Finally, the Bogoliubov coefficients and the analog of the self-consistent equations (19) and (20) now read

$$
\begin{gathered}
\bar{u}_{\mathbf{k}}^{2}, \bar{v}_{\mathbf{k}}^{2}= \pm 1 / 2+\bar{A}_{\mathbf{k}} / 2 \bar{\omega}_{\mathbf{k}}, \quad \bar{u}_{\mathbf{k}} \bar{v}_{\mathbf{k}}=\bar{B}_{\mathbf{k}} / 2 \bar{\omega}_{\mathbf{k}}, \\
\mu=-\frac{3 J^{\prime}}{4}+\frac{3}{N_{0}} \sum_{\mathbf{k}} B_{\mathbf{k}} \bar{v}_{\mathbf{k}}\left(\bar{v}_{\mathbf{k}}-\bar{u}_{\mathbf{k}}\right), \\
N_{0}=1-\frac{3}{N^{\prime}} \sum_{\mathbf{k}} \bar{v}_{\mathbf{k}}^{2} .
\end{gathered}
$$

Note that $B_{\mathbf{k}}$ (not $\bar{B}_{\mathbf{k}}$ ) enters the momentum summation in Eq. (28) (for details, see Appendix C). In addition to $\mu$ and $N_{0}$, now $A_{1,2,3}^{\mathrm{HF}}$ and $B_{1,2,3}^{\mathrm{HF}}$ are also self-consistently calculated. The set of equations (23)-(29) constitutes the HF approximation.

The resulting triplon dispersion is included in Fig. 4, which shows that corrections arising from $\mathcal{H}_{4}^{\mathrm{HF}}$ are small, except for a slight upward renormalization of the gap. For a fixed $J^{\prime}$, the minimum wave vector $\mathbf{Q}_{0}$ displays the same qualitative behavior in terms of $J^{\prime \prime}$ as found in the harmonic approximation; see Fig. 5. In the incommensurate regime, $\mathbf{Q}_{0}$ numerically deviates from the harmonic result for small $J^{\prime}$ (but recovers the harmonic results in the large- $J^{\prime}$ limit); see Fig. 6. Moreover, the line marking the CIT is slightly shifted as well; see Fig. 5.

Figure 7 shows the evolution of $N_{0}$ as a function of $J^{\prime}$ for fixed $J^{\prime \prime}$, again comparing the HF results to those from the harmonic approximation. The deviations are small in general and vanish in the strong-dimer limit $J^{\prime} \rightarrow \infty$. Inside the disordered phase, we observe $N_{0}>0.7$, which implies that $v_{\mathbf{k}}$ and $\bar{v}_{\mathbf{k}} \lesssim 0.1$; see Eqs. (20) and (29). The fact that $\bar{v}_{\mathbf{k}}$ is

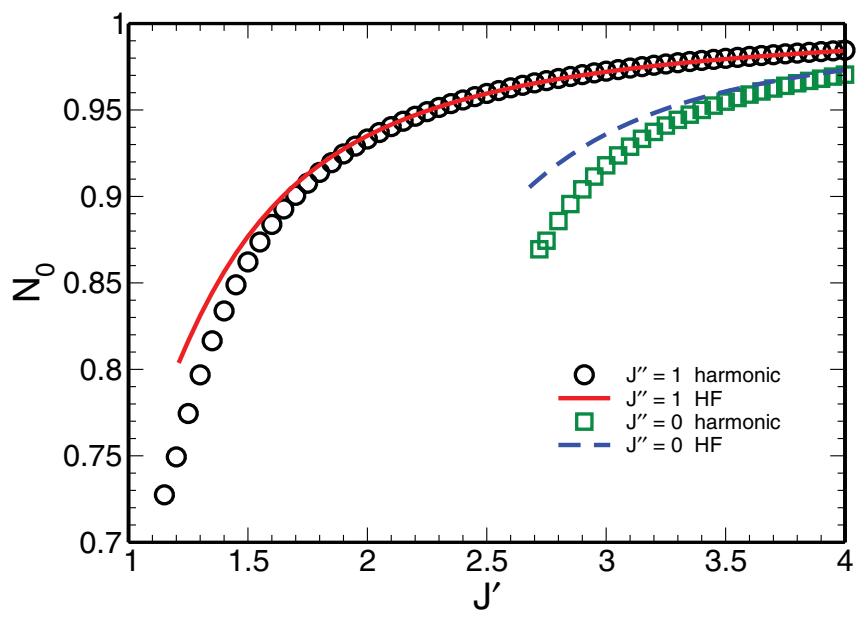

FIG. 7. (Color online) Parameter $N_{0}$ as a function of $J^{\prime}$ for the lines $J^{\prime \prime}=0$ and $J^{\prime \prime}=1$ at the harmonic and Hartree-Fock levels. a small quantity is used in order to derive the self-consistent equations (28) and (29); see Appendix C for details.

We note that we experienced more difficulties in obtaining self-consistent solutions near the phase boundary in the HF approximation as compared to the harmonic one, but these problems are cured upon including the cubic term; see Sec. IV B below.

\section{B. Hartree-Fock-cubic approximation}

We implement a Hartree-Fock-cubic (HF-cubic) approximation by perturbatively adding the effect of $\mathcal{H}_{3}$ to the mean-field Hamiltonian $\mathcal{H}=E_{0}+E_{0}^{\mathrm{HF}}+\mathcal{H}_{2}+\mathcal{H}_{4}^{\mathrm{HF}}$. This scheme, which simplifies the treatment of the cubic triplet interaction considerably, is similar in spirit to the one adopted in Refs. 37 and 39, where an interacting Hamiltonian for triplets without the cubic term is treated self-consistently first, and then the lowest-order corrections due to the cubic term are added.

Using the Bogoliubov transformation (14) with the renormalized coefficients $\bar{u}_{\mathbf{k}}$ and $\bar{v}_{\mathbf{k}}(27)$, one can show that $\mathcal{H}_{3}(8)$ in terms of the $b_{\mathbf{k} \alpha}^{\dagger}$ and $b_{\mathbf{k} \alpha}$ operators reads

$$
\begin{aligned}
\mathcal{H}_{3}= & \frac{1}{2 \sqrt{N^{\prime}}} \sum_{\mathbf{k}, \mathbf{p}} \sum_{\alpha, \beta, \gamma}^{\prime} \Gamma_{1}(\mathbf{k}, \mathbf{p})\left(b_{\mathbf{k}-\mathbf{p} \alpha}^{\dagger} b_{\mathbf{p} \beta}^{\dagger} b_{\mathbf{k} \gamma}+\text { H.c. }\right) \\
& +\frac{1}{2 \sqrt{N^{\prime}}} \sum_{\mathbf{k}, \mathbf{p}} \Gamma_{2}(\mathbf{k}, \mathbf{p})\left(b_{\mathbf{k}-\mathbf{p} x}^{\dagger} b_{\mathbf{p} y}^{\dagger} b_{-\mathbf{k} z}^{\dagger}+\text { H.c. }\right) .
\end{aligned}
$$

Here the sum over $\alpha, \beta, \gamma$ has only three components: $(\alpha, \beta, \gamma)=(x, y, z),(z, x, y)$, and $(y, z, x)$. The renormalized vertex $\Gamma_{1}(\mathbf{k}, \mathbf{p})$ is given by

$$
\begin{aligned}
\Gamma_{1}(\mathbf{k}, \mathbf{p})= & \left(\xi_{\mathbf{k}-\mathbf{p}}-\xi_{\mathbf{p}}\right)\left(\bar{u}_{\mathbf{k}-\mathbf{p}} \bar{u}_{\mathbf{p}} \bar{u}_{\mathbf{k}}+\bar{v}_{\mathbf{k}-\mathbf{p}} \bar{v}_{\mathbf{p}} \bar{v}_{\mathbf{k}}\right) \\
& +\left(\xi_{\mathbf{k}}+\xi_{\mathbf{p}}\right)\left(\bar{v}_{\mathbf{k}-\mathbf{p}} \bar{u}_{\mathbf{p}} \bar{v}_{\mathbf{k}}+\bar{u}_{\mathbf{k}-\mathbf{p}} \bar{v}_{\mathbf{p}} \bar{u}_{\mathbf{k}}\right) \\
& -\left(\xi_{\mathbf{k}-\mathbf{p}}+\xi_{\mathbf{k}}\right)\left(\bar{v}_{\mathbf{k}-\mathbf{p}} \bar{u}_{\mathbf{p}} \bar{u}_{\mathbf{k}}+\bar{u}_{\mathbf{k}-\mathbf{p}} \bar{v}_{\mathbf{p}} \bar{v}_{\mathbf{k}}\right)
\end{aligned}
$$

with $\xi_{\mathrm{p}}$ being the bare cubic vertex (12), and the vertex $\Gamma_{2}(\mathbf{k}, \mathbf{p})=-\Gamma_{1}(\mathbf{k}, \mathbf{p})$ in addition to the replacements $\bar{u}_{\mathbf{k}} \leftrightarrow$ $\bar{v}_{\mathbf{k}}$. The vertices $\Gamma_{1}(\mathbf{k}, \mathbf{p})$ and $\Gamma_{2}(\mathbf{k}, \mathbf{p})$ are illustrated in Fig. 8(a).

The lowest-order diagrams that contribute to the (normal) self-energy $\Sigma_{3}(\mathbf{k}, \omega)$ are shown in Figs. 8(b)-8(e). In each diagram, the solid line corresponds to the bare $b$ triplon propagator (we now omit the index $\alpha$ since the three triplon branches are degenerate)

$$
G_{0}^{-1}(\mathbf{k}, \omega)=\omega-\bar{\omega}_{\mathbf{k}}+i \delta .
$$

Note here that no anomalous bare $b$ propagators exist; in principle, those are generated in perturbation theory, but will be neglected in the following. ${ }^{40}$ Using standard diagrammatic techniques for bosons at zero temperature, one shows that only the diagrams $(b)$ and $(e)$ are finite, and therefore $\Sigma_{3}(\mathbf{k}, \omega)=$ $\Sigma_{3}^{(b)}(\mathbf{k}, \omega)+\Sigma_{3}^{(e)}(\mathbf{k}, \omega)$ with

$$
\begin{aligned}
& \Sigma_{3}^{(b)}(\mathbf{k}, \omega)=\frac{1}{4 N^{\prime}} \sum_{\mathbf{q}} \frac{\Gamma_{1}^{2}(\mathbf{k}, \mathbf{q})}{\omega-\bar{\omega}_{\mathbf{q}}-\bar{\omega}_{\mathbf{k}-\mathbf{q}}+i \delta}, \\
& \Sigma_{3}^{(e)}(\mathbf{k}, \omega)=-\frac{1}{4 N^{\prime}} \sum_{\mathbf{q}} \frac{\Gamma_{2}^{2}(\mathbf{k}, \mathbf{q})}{\omega+\bar{\omega}_{\mathbf{q}}+\bar{\omega}_{\mathbf{k}-\mathbf{q}}-i \delta} .
\end{aligned}
$$


(a)

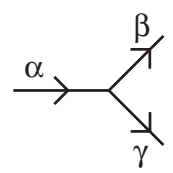

(1)

(b)

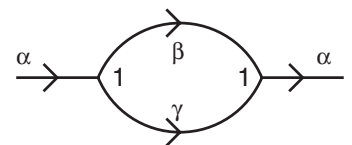

(d)

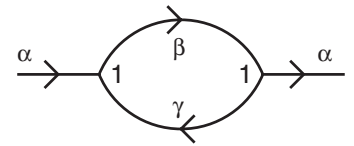

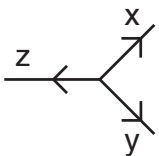

(2)

(c)

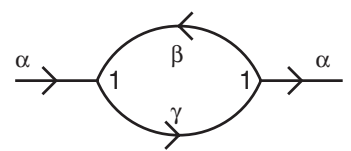

(e)

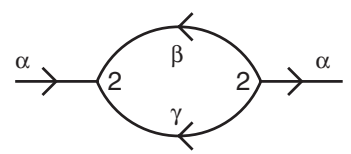

FIG. 8. (a) Renormalized cubic vertices $\Gamma_{1}(\mathbf{k}, \mathbf{p})$, (1), and $\Gamma_{2}(\mathbf{k}, \mathbf{p}),(2)$. Here $(\alpha, \beta, \gamma)=(x, y, z),(z, x, y)$, and $(y, z, x)$. (b)-(e) Lowest-order diagrams resulting from the combination of vertices (1) and (2) that contribute to the normal self-energy $\Sigma_{3}(\mathbf{k}, \omega)$.

The renormalized triplon energy $\Omega_{\mathbf{k}}$ is given by the poles of the full Green's function $G(\mathbf{k}, \omega)$, i.e,

$$
G^{-1}(\mathbf{k}, \omega)=\omega-\bar{\omega}_{\mathbf{k}}-\Sigma_{3}(\mathbf{k}, \omega)=0 .
$$

We solve Eq. (34) within the so-called off-shell approximation, which consists in evaluating the self-energy at $\omega=\Omega_{\mathbf{k}}-i \tilde{\Gamma}_{\mathbf{k}}$, i.e.,

$$
\Omega_{\mathbf{k}}-i \tilde{\Gamma}_{\mathbf{k}}-\bar{\omega}_{\mathbf{k}}-\Sigma_{3}\left(\mathbf{k}, \Omega_{\mathbf{k}}-i \tilde{\Gamma}_{\mathbf{k}}\right)=0 .
$$

[Recall that in the more common on-shell approximation, the self-energy is evaluated at the bare single-particle energy, i.e., $\Omega_{\mathbf{k}}-i \tilde{\Gamma}_{\mathbf{k}}-\bar{\omega}_{\mathbf{k}}-\Sigma_{3}\left(\mathbf{k}, \bar{\omega}_{\mathbf{k}}\right)=0$.] Moreover, instead of using Eq. (35), causality requires us to consider

$$
\Omega_{\mathbf{k}}-i \tilde{\Gamma}_{\mathbf{k}}-\bar{\omega}_{\mathbf{k}}-\Sigma_{3}\left(\mathbf{k}, \Omega_{\mathbf{k}}+i \tilde{\Gamma}_{\mathbf{k}}\right)=0
$$

(a)

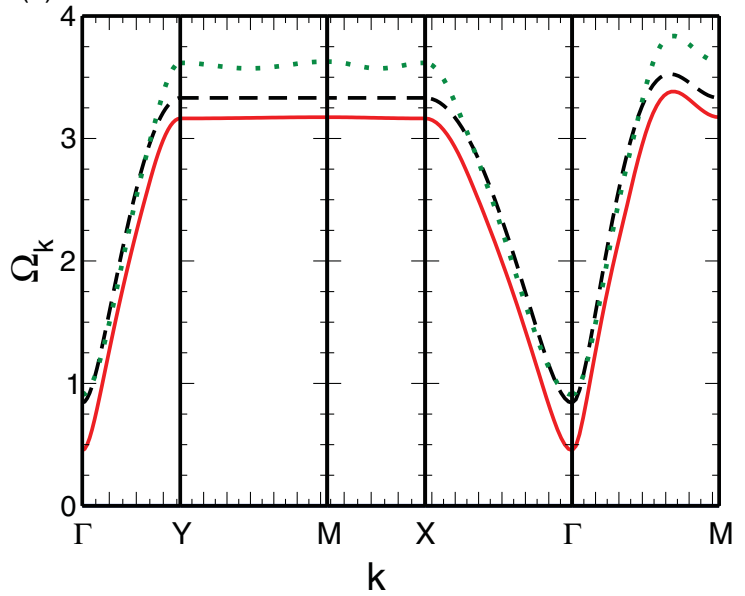

(b)

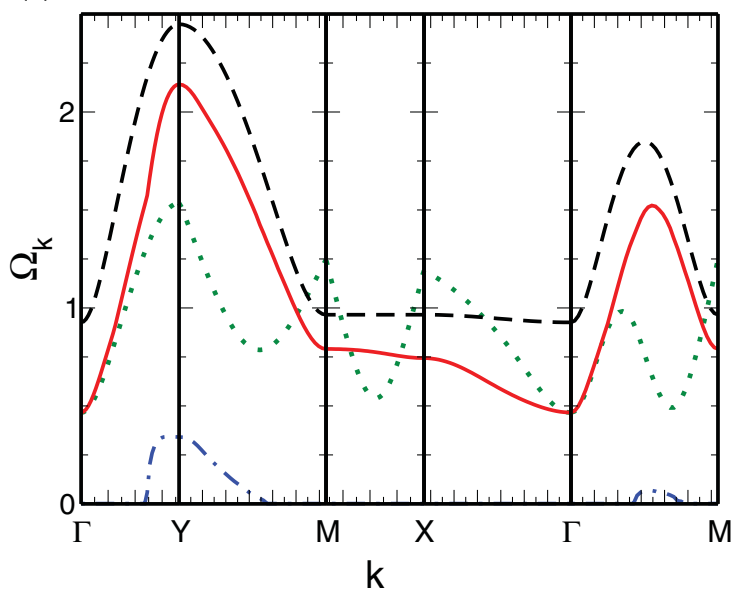

The procedure outlined above follows Ref. 35, where spin-wave excitations of the isotropic triangular-lattice $\mathrm{AF}$ Heisenberg model $\left(J^{\prime}=J^{\prime \prime}=1\right)$ were calculated. It is shown by Chernyshev et al. ${ }^{35}$ that the on-shell approximation leads to discontinuities in the spin-wave spectrum and concomitant logarithmic singularities in the decay rate $\tilde{\Gamma}_{\mathbf{k}}$, and that the off-shell approximation regularizes such singularities. Finally, the replacement $\Omega_{\mathbf{k}}-i \tilde{\Gamma}_{\mathbf{k}} \rightarrow \Omega_{\mathbf{k}}+i \tilde{\Gamma}_{\mathbf{k}}$ in the argument of the self-energy guarantees that the quasiparticle pole is in the correct half of the complex plane (we refer the reader to Appendix D, Ref. 35, for details).

In Fig. 9, we compare the renormalized triplon energy $\Omega_{\mathbf{k}}$ obtained in the HF-cubic approximation to that from HF. Clearly, the inclusion of the cubic term leads to sizable changes of the dispersion, which are particularly pronounced in the incommensurate regime [see Fig. 9(b) for $J^{\prime}=1.5$ and $\left.J^{\prime \prime}=1\right]$. For instance, the dispersion along $\Gamma-\mathbf{M}$ is significantly enhanced by the cubic term. Most notably, the triplon gap $\Delta$ is renormalized downward; see Fig. 10 below. In other words, cubic interactions tend to destabilize the paramagnetic phase, whereas (repulsive) quartic interactions have the opposite effect.

The behavior of the minimum wave vector $\mathbf{Q}_{0}$ as a function of $J^{\prime \prime}$ is again qualitatively similar to the harmonic one, Fig. 5. Interestingly, the shift of the CIT line due to the cubic interaction is opposite to (and larger than) that induced by the quartic interaction, such that the CIT line is now located at some $J_{\text {CIT }}^{\prime \prime}>0.75$, which moreover depends nonmonotonically on $J^{\prime}$, Fig. 3. Figure 9 also displays the decay rate of triplons caused by two-particle decay via $\mathcal{H}_{3}-$ this is nonzero whenever the single-particle branch is above the two-particle threshold.

We conclude that the cubic triplon term is of vital importance for a quantitative description of the triplon dynamics in the frustrated coupled-dimer model (1), especially in the incommensurate regime. This is in contrast to, e.g., the unfrustrated asymmetric bilayer model studied in Ref. 37

FIG. 9. (Color online) Triplon dispersion relation along some particular lines of the dimerized Brillouin zone for (a) $J^{\prime}=3$ and $J^{\prime \prime}=0$ and (b) $J^{\prime}=1.5$ and $J^{\prime \prime}=1$ at the HF (dashed black line) and HF-cubic (solid red line) approximations. The triplon decay rate $\tilde{\Gamma}_{\mathbf{k}}$ (dot-dashed blue line) and the bottom of the two-particle continuum (dotted green line) both at the HF-cubic approximation are also shown. The $A-B$ line includes the triplon minimum energy of the corresponding approximation. 
where the cubic term leads to minor corrections only. A further discussion of the physical implications of our results is given below.

\section{DISCUSSION}

\section{A. Phase boundary}

For the different levels of approximation, the evolution of the energy gap $\Delta$ with the dimerization strength $J^{\prime}$ is shown in Fig. 10 for $J^{\prime \prime}=0,0.8$, and 1 . As expected, $\Delta$ increases with $J^{\prime}$; the vanishing of the gap defines a critical value $J_{c}^{\prime}$ where the singlet phase becomes unstable toward magnetic order. Assuming a continuous QPT, we fit the data to

$$
\Delta=a_{0}+a_{1} J^{\prime}+a_{2} \frac{1}{J^{\prime}}+a_{3}\left(J^{\prime}\right)^{2}
$$

and we use the condition $\Delta=0$ to estimate the critical coupling $J_{c}^{\prime}$. (Note that the present approximations cannot be expected to yield nontrivial critical exponents. Omitting the $1 / J^{\prime}$ fitting term leads to only minor changes of $J_{c}^{\prime}$.)

The resulting phase boundary for $0<J^{\prime \prime}<1.5$ is shown in Fig. 3, with the HF-cubic result ( $\square$ ) being our best approximation. The phase diagram in Fig. 3 displays four distinct regions: At large $J^{\prime}$ we have the gapped dimer phase, with spin correlations peaked at $\mathbf{Q}_{0}=(0,0)$ for $0 \leqslant J^{\prime \prime} \leqslant J_{\text {CIT }}^{\prime \prime}$ and incommensurate $\mathbf{Q}_{0}$ for $J^{\prime \prime}>J_{\text {CIT }}^{\prime \prime}$. At the harmonic level, $J_{\text {CIT }}^{\prime \prime}$ equals 0.75 but acquires a $J^{\prime}$ dependence from anharmonic terms; see Figs. 5 and 6. The closing of the triplon gap at $J_{c}^{\prime}$ for a given $J^{\prime \prime}$ leads to magnetic long-range order at wave vector $\mathbf{Q}_{0}$ (provided that no other phase intervenes). One concludes that the system displays collinear Néel order for $J<J_{\text {CIT }}^{\prime \prime}$ and noncollinear spiral order for $J^{\prime \prime}>J_{\text {CIT. As }}^{\prime \prime}$ indicated in Fig. 2, an additional paramagnetic phase might be realized near the crossing of the CIT and the order-disorder transition lines, ${ }^{9}$ with a more detailed discussion given in Sec. V B.

Let us discuss quantitatively the evolution for two values of $J^{\prime \prime}$. (i) $J^{\prime \prime}=0$ : Here we find a critical coupling $J_{c}^{\prime}=2.65$ (2.76) at the harmonic (HF-cubic) level, which is quite close to the one determined via QMC simulations for the (topologically equivalent) staggered dimerized AF Heisenberg model on a square lattice, $J_{c}^{\prime \text { QMC }}=2.5196 .{ }^{33}$ For $J^{\prime}<J_{c}^{\prime}$, the system indeed develops collinear Néel order with $\mathbf{Q}_{0}=(0,0) .^{33,34}$ (ii) $J^{\prime \prime}=1$, where we find $J_{c}^{\prime}=1.09$ (1.34) at the harmonic (HF-cubic) level. Again, this is a very reasonable result since LRO is certainly present at the isotropic point $\left(J^{\prime}=J^{\prime \prime}=1\right)$. One difference from case (i) is that the ordering wave vector varies with $J^{\prime}$ inside the ordered phase (see Appendix A for the corresponding classical-limit results); therefore, $\mathbf{Q}_{0}$ at the phase boundary is distinct from the Goldstone wave vectors $\mathbf{K}, \mathbf{K}^{\prime}$ of the $120^{\circ}$ structure; see also Fig. 1(c).

\section{B. Commensurate-incommensurate transition}

As shown above, the minimum wave vector $\mathbf{Q}_{0}$ of the triplon dispersion in the gapped paramagnetic phase of the Heisenberg model (1) is locked to $(0,0)$ for small $J^{\prime \prime}$, while it moves to incommensurate values for larger $J^{\prime \prime}$ (Figs. 5 and 6), with the boundary being located near $J_{\text {CIT }}^{\prime \prime} \lesssim 0.75$. This CIT, driven by increasing magnetic frustration, has various consequences.

Right at the CIT, the quadratic piece of the triplon dispersion vanishes in one of the two space directions (independent of the level of approximation), i.e., we find for the triplon propagator

$$
G^{-1}(\mathbf{k}, \omega)=-\omega^{2}+\Delta^{2}+c_{1} \tilde{k}_{1}^{2}+d_{1} \tilde{k}_{2}^{4}
$$

in a small-momentum expansion around the $\Gamma$ point, where $\tilde{k}_{1,2}$ are the two components of $\mathbf{k}$ perpendicular and parallel to the $\mathbf{Q}_{0}$ that is taken beyond the CIT. Moreover, in the incommensurate regime near the CIT, the dispersion along the $\tilde{k}_{2}$ direction (connecting $\Gamma$ and $\mathbf{Q}_{0}$ ) is anomalously flat. This is illustrated in Fig. 11, which shows a contour plot of the triplon dispersion $\Omega_{\mathbf{k}}$ (HF-cubic approximation) for $J^{\prime}=1.5$ and $J^{\prime \prime}=0.8$. Qualitatively, the soft dispersion near the CIT will lead to an effective dimensional reduction. When the triplon gap closes, $\Delta=0$, Eq. (38) defines a quantum Lifshitz point; see Sec. V D below.

Of course, the physics underlying the CIT is also observed inside the ordered phase. This has been studied in particular in the nondimerized case, $J^{\prime}=1$. Linear spin-wave theory ${ }^{7,8}$ yields a QPT from collinear Néel to spiral order at $J_{c}^{\prime \prime}=0.5$, where the ordering wave vector changes from a commensurate to an incommensurate value. (The analysis of the classical ground state for the general case $J^{\prime} \neq 1$ is given in Appendix A.) The magnetization curve has a minimum at the transition, where the spin-wave velocity vanishes along one particular direction in $k$ space. Coupled-cluster calculations ${ }^{10}$ find a
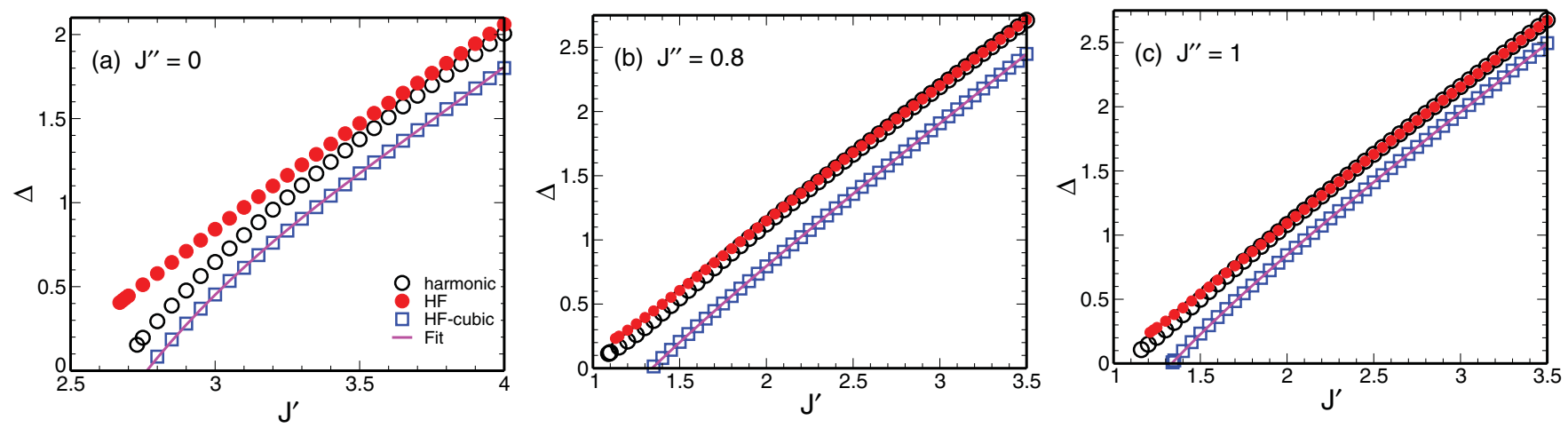

FIG. 10. (Color online) Triplon gap $\Delta$ as a function of $J^{\prime}$ for (a) $J^{\prime \prime}=0$, (b) $J^{\prime \prime}=0.8$, and (c) $J^{\prime \prime}=1$ at the harmonic (O), HF (๑), and HF-cubic ( $\square$ ) approximations. The solid line is a fit to the data using Eq. (37). 


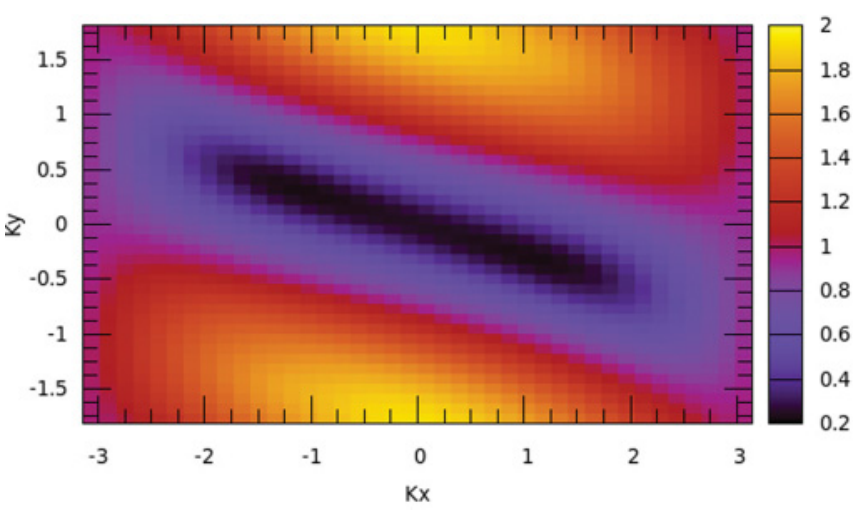

FIG. 11. (Color online) Contour plot of the triplon energy $\Omega_{\mathbf{k}}$ (HF-cubic) for $J^{\prime}=1.5$ and $J^{\prime \prime}=0.8$, i.e., near the CIT.

scenario similar to spin-wave theory, but here $J_{c}^{\prime \prime} \approx 0.8$. Finally, series-expansion results ${ }^{9}$ not only indicate a CIT at $J^{\prime \prime} \approx 0.75$, but they also provide evidence for a disordered phase in the vicinity of the CIT, i.e, for $0.68<J^{\prime \prime}<0.91$.

\section{Two-particle decay}

The cubic triplon term $\mathcal{H}_{3}$ is generically present in the model (1) and thus enables two-particle decay of triplons. ${ }^{30,31}$ Such decay occurs if the one-triplon dispersion moves inside the two-particle continuum.

As can be seen from Fig. 9, two-particle decay does not happen in the commensurate regime of small $J^{\prime \prime}$, where the one-particle spectrum is always below the two-particle continuum. Note that an exceptional case is realized right at the QPT to the magnetically ordered state, where the lower bound of the two-particle continuum coincides with the single-particle branch near the ordering wave vector $(0,0)$. This nontrivial coupling has been argued to lead to a novel universality class for the phase transition; ${ }^{34}$ see also Sec. VD below.

The situation is different in the incommensurate regime, where high-energy triplons can decay into triplon pairs; see Fig. 9 for the calculated decay rate. Note that such decay never happens near $\mathbf{Q}_{0}$, i.e., cubic interactions are not part of the critical theory in the incommensurate case.

As an aside, we note that decay of high-energy modes also occurs inside the ordered phase, where spin waves can decay into pairs of spin waves provided that the order is noncollinear; for details, see Ref. 35.

\section{Quantum criticality}

Let us briefly discuss the quantum phase transitions in the phase diagram shown in Fig. 2.

The transition from the dimer phase to the collinear LRO state with $\mathbf{Q}_{0}=(0,0)$ would in principle be expected to be in the standard Heisenberg [or $\mathrm{O}(3)$ ] universality class in $D=$ $2+1$ dimensions; however, the cubic triplon term becomes part of the critical theory and leads to a new universality class (labeled class B in Ref. 34), with leading $\mathrm{O}(3)$ exponents and anomalously large corrections to scaling. ${ }^{33,34}$
The transition from the dimer phase to the noncollinear LRO state does not display this complication and therefore is a standard $\mathrm{O}(N)$ QPT in $D=2+1$, with $N=4$ (assuming a single transition to coplanar spiral order).

Finally, we can discuss the multicritical point where the collinear LRO, noncollinear LRO, and dimer phases meet. Here, the softness of the triplon dispersion in one direction, Eq. (38), implies that the quadratic piece of the critical field theory takes the form

$$
\mathcal{S}=\int d^{2} x d \tau \phi_{a}\left(\delta+\partial_{\tau}^{2}+\partial_{x}^{2}+\partial_{y}^{4}\right) \phi_{a},
$$

where $\phi_{a}$ are the components of the magnetic order parameter, and $\delta=0$ defines the phase transition point. Such $(d, m)$ quantum Lifshitz points (here $d=2$, and $m=1$ refers to the number of "soft" directions) have been discussed before, ${ }^{28,29}$ but the relevant case of $d=2$ dimensions with undamped order-parameter dynamics has not been studied in any detail. A thorough analysis of this critical theory is therefore deferred to a future publication; here we only make a few qualitative remarks. The absence of the quadratic derivative in the $y$ direction implies that the effective dimensionality is reduced compared to a standard $\phi^{4}$ theory, or in other words, the upper critical dimension (in the quantum case) is increased from $d_{c}^{+}=3$ to $d_{c}^{+}=3+m / 2$ (Ref. 29). While Eq. (39), supplemented by a standard quartic term (note that a cubic term $^{34}$ might be important as well), describes a continuous multicritical point, it is conceivable that this is preempted by a fluctuation-induced transition into a novel phase. We speculate that this could be a nontrivial paramagnetic phase (e.g., with a symmetry-breaking dimerization), as deduced for $J^{\prime}=1$ from series-expansion studies. ${ }^{9}$

\section{E. Application to $\operatorname{EtMe}_{3} \mathrm{P}\left[\operatorname{Pd}(\mathrm{dmit})_{2}\right]_{2}$}

As mentioned in the Introduction, the experimental findings ${ }^{21-24}$ indicate that the organic Mott insulator $\mathrm{EtMe}_{3} \mathrm{P}\left[\mathrm{Pd}(\mathrm{dmit})_{2}\right]_{2}$ realizes a columnar VBS phase at low temperature and pressure. According to Ref. 22, this system can be described by the Heisenberg model (1) with $J^{\prime}=1$ and $J^{\prime \prime}=1.05$. Although this configuration is outside of the VBS region predicted by our bond-operator analysis, it is quite close to the VBS phase boundary (see the orange diamond, Fig. 3). It is conceivable that a combination of longer-range or ring-exchange interactions, which arise in proximity to the Mott transition, ${ }^{6}$ increases the level of magnetic frustration, which is then released by a lattice dimerization through magnetoelastic couplings (note that organic compounds of the $X\left[\operatorname{Pd}(\mathrm{dmit})_{2}\right]_{2}$ display a rather soft lattice). As a result, a VBS phase can emerge, whose magnetic couplings are explicitly dimerized as in our Hamiltonian (1).

Therefore, we believe that the excitation spectrum of the organic compound may display features qualitatively similar to the ones shown, e.g., in Fig. 9(b), which corresponds to a configuration $\left(J^{\prime}=1.5, J^{\prime \prime}=1\right)$ close to the critical line. Hence we predict the spin correlations in the VBS phase of $\mathrm{EtMe}_{3} \mathrm{P}\left[\mathrm{Pd}(\mathrm{dmit})_{2}\right]_{2}$ to be incommensurate, which may be checked in future neutron scattering or NMR experiments. Moreover, it would be interesting to see whether one could 
drive the material into a state with magnetic LRO by moving farther away from the Mott transition (and thus reducing the influence of magnetic couplings beyond nearest-neighbor exchange).

\section{SUMMARY}

We have studied a dimerized Heisenberg AF on a spatially anisotropic triangular lattice, with a focus on its quantum paramagnetic (i.e., dimer) phase. Starting from bond-operator mean-field theory, we have included interaction corrections to the harmonic approximation at the Hartree-Fock level for the quartic term and in second-order perturbation theory for the cubic term. We have shown that this Hartree-Fock-cubic approximation gives sensible results for the triplon dispersion for the investigated part of the parameter space (away from the one-dimensional limit). The resulting boundary of the dimer phase where the triplon gap closes, Fig. 3, is one of our main results; its location in the unfrustrated limit $J^{\prime \prime}=0$ is in good quantitative agreement with QMC data.

The minimum wave vector $\mathbf{Q}_{0}$ of the triplon spectrum displays a commensurate-incommensurate transition as frustration is increased. At this CIT, the quadratic piece of the triplon dispersion vanishes in one momentum direction. The closing of the triplon gap at the CIT leads to a quantum Lifshitz point, the detailed study of which is left for future work: it either leads to distinct power-law critical behavior or it may even be inherently unstable, leading to a novel intermediate phase, Fig. 2. Remarkably, we find the critical $J^{\prime}$ to be only slightly above unity, suggesting that the origin of the nontrivial VBS phase seen in series-expansion studies ${ }^{9}$ for the nondimerized model is indeed the enhanced fluctuations near the quantum Lifshitz point. Finally, in the incommensurate regime of the dimer phase, the cubic triplon interaction leads to two-particle decay of triplons at high energies.

Our study paves the way for further investigations of frustrated dimerized magnets. As those are difficult to access using QMC simulations, due to the inherent minus sign problem, bond-operator as well as series-expansion techniques are often the methods of choice. Clearly, the inclusion of longer-range and cyclic exchange interactions on triangular and Kagome lattice geometries would be most interesting. On the methodological side, a self-consistent treatment of the cubic term might further improve the numerical accuracy. In addition, the formation of bound states of triplons-which would signify the instability toward phases other than those with magnetic LRO_-should be studied; we leave this for future work. Finally, the interplay of magnetoelastic couplings and longer-range exchange interactions should be studied near the isotropic point, with an eye toward $\mathrm{EtMe}_{3} \mathrm{P}\left[\mathrm{Pd}(\mathrm{dmit})_{2}\right]_{2}$.

\section{ACKNOWLEDGMENTS}

We thank L. Fritz, M. Garst, and T. Vojta for helpful discussions. This research was partially supported by the DFG through SFB 608 (Köln), GRK 1621 (Dresden), and FOR 960. R.L.D. also acknowledges support by FAPESP (project No. 10/00479-6).

\section{APPENDIX A: CLASSICAL PHASE DIAGRAM}

To determine the classical phases of the model (1), we parametrize the spins $\mathbf{S}_{i}^{1}$ and $\mathbf{S}_{i}^{2}$ [Fig. 1(a)] according to

$$
\begin{aligned}
& \mathbf{S}_{i}^{1}=\hat{e}_{1} \cos \left(\mathbf{Q} \cdot \mathbf{R}_{i}\right)+\hat{e}_{2} \sin \left(\mathbf{Q} \cdot \mathbf{R}_{i}\right), \\
& \mathbf{S}_{i}^{2}=\hat{e}_{3} \cos \left(\mathbf{Q} \cdot \mathbf{R}_{i}\right)+\hat{e}_{4} \sin \left(\mathbf{Q} \cdot \mathbf{R}_{i}\right),
\end{aligned}
$$

which assumes coplanar order. Here $\hat{e}_{i}$ are a set of unit vectors that obey $\hat{e}_{1} \cdot \hat{e}_{2}=\hat{e}_{3} \cdot \hat{e}_{4}=0, \hat{e}_{1} \cdot \hat{e}_{3}=\hat{e}_{2} \cdot \hat{e}_{4}=\cos \theta$, and $\hat{e}_{2} \cdot \hat{e}_{3}=-\hat{e}_{1} \cdot \hat{e}_{4}=\sin \theta$. Substituting Eq. (A1) into the Hamiltonian (4), it is easy to see that the total energy is $E=(N / 2) S^{2} J_{\mathbf{Q}}(\theta)$, with

$$
\begin{aligned}
J_{\mathbf{Q}}(\theta)= & J^{\prime} \cos \theta+2 J^{\prime \prime} \cos \left(Q_{x}\right)+\cos \left(Q_{x}+\theta\right) \\
& +\cos \left(\sqrt{3} Q_{y}+\theta\right)+\cos \left(Q_{x}+\sqrt{3} Q_{y}+\theta\right) .
\end{aligned}
$$

For fixed $J^{\prime}$ and $J^{\prime \prime}$, the ground-state energy is determined by minimizing Eq. (A2) with respect to the components of the vector $\mathbf{Q}$ and the angle $\theta$.

We find two phases, Fig. 12: (a) collinear order, with a commensurate ordering wave vector $\mathbf{Q}=(0,0)$ and $\theta=\pi$, realized for $J^{\prime \prime} \leqslant J_{\text {CIT }}^{\prime \prime}\left(J^{\prime}\right)$, and (b) noncollinear order, with incommensurate $\mathbf{Q}=\left(Q_{\mathrm{xm}}, Q_{\mathrm{ym}}\right)$ and $\theta=\pi-Q_{\mathrm{xm}} / 2-\sqrt{3} Q_{\mathrm{ym}}$. The components of $\mathbf{Q}$ solve

$$
\begin{aligned}
\mu \sin \left(Q_{x} / 2\right) \cos \left(\sqrt{3} Q_{y}\right)-\sin \left(Q_{x} / 2-\sqrt{3} Q_{y}\right) & =0, \\
4 J^{\prime \prime} \cos \left(Q_{x} / 2\right)-\mu \cos \left(\sqrt{3} Q_{y}\right) & =1,
\end{aligned}
$$

with $\mu=2 J^{\prime} /\left(1+J^{\prime}\right)$. Numerical solutions of (A3) can be easily obtained, which yield the phase boundary in Fig. 12.

Interestingly, it is possible to solve Eqs. (A3) analytically for the particular cases $J^{\prime}=0,1$, and $\infty$, which, respectively, correspond to $\mu=0,1$, and 2 . The solutions can be written in the following way:

$$
\begin{aligned}
& Q_{\mathrm{xm}}=2 \arccos \left(\frac{1-\mu^{2}+2 \mu}{4 J^{\prime \prime}-\mu(\mu-1)}\right), \\
& Q_{\mathrm{ym}}=\frac{\lambda_{\mu}}{\sqrt{3}} \arccos \left(\frac{1-\mu^{2}+2 \mu}{4 J^{\prime \prime}-\mu(\mu-1)}\right) .
\end{aligned}
$$

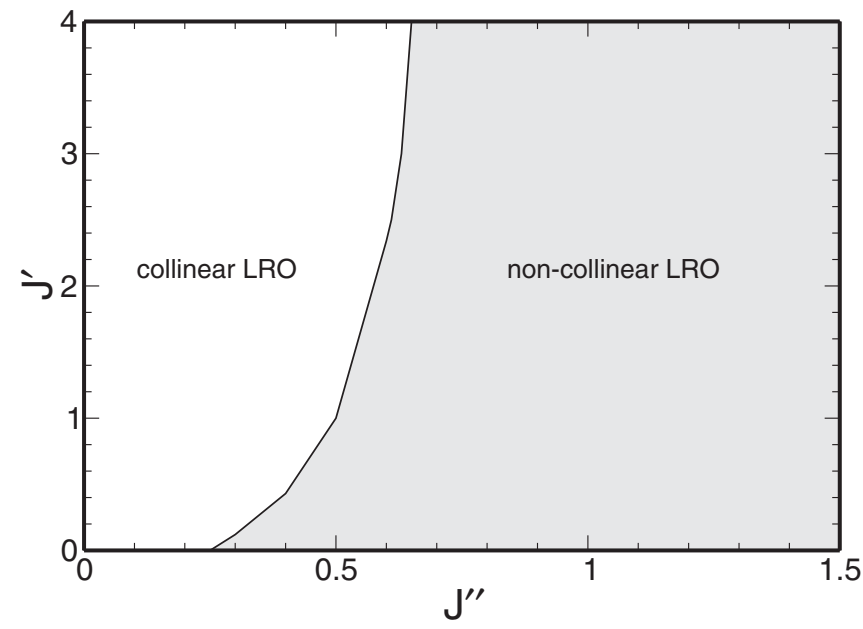

FIG. 12. Classical phase diagram for the Heisenberg model (1), obtained from numerically solving Eq. (A3). 
Here, $\lambda_{0}=-\lambda_{2}=1$ and $\lambda_{1}=0$. Since the argument of $\arccos (x)$ is within the range $-1 \leqslant x \leqslant 1$, we conclude that the noncollinear phase is stable for $J^{\prime \prime} \geqslant J_{\text {CIT }}^{\prime \prime}$, where

$$
J_{\mathrm{CIT}}^{\prime \prime}=\frac{1}{4}+\frac{J^{\prime}}{2\left(1+J^{\prime}\right)},
$$

valid for $J^{\prime}=0,1$, and $\infty$. (For $J^{\prime}=\infty$, we obtain $J_{\text {CIT }}^{\prime \prime}=$ 0.75 and $Q_{\mathrm{xm}, \mathrm{ym}}=q_{\mathrm{xm}, \mathrm{ym}}$, i.e., the classical result matches the corresponding features of the bond-operator result for $S=$ 1/2.)

Quantum corrections to the classical phase boundary can be obtained using nonlinear spin-wave theory. For $J^{\prime}=1$, this has recently been demonstrated to move the CIT boundary to $J^{\prime \prime}=0.77$ at order $1 / S$ (Ref. 41$)$.

\section{APPENDIX B: EFFECTIVE TRIPLET HAMILTONIAN IN REAL SPACE}

Here we quote the full expressions for the real-space Hamiltonian (6), which can be obtained using straightforward algebra via Eq. (3),

$$
\begin{aligned}
\mathcal{H}_{0}= & -\frac{3 J^{\prime}}{4} \sum_{j} s_{j}^{\dagger} s_{j} \\
\mathcal{H}_{2}= & \frac{J^{\prime}}{4} \sum_{j} t_{j \alpha}^{\dagger} t_{j \alpha} \\
& +\frac{1}{4} \sum_{j, n} g_{2}(n)\left[\left(s_{j} s_{j+n}^{\dagger} t_{j \alpha}^{\dagger} t_{j+n \alpha}+\text { H.c. }\right)\right. \\
& \left.+\left(s_{j}^{\dagger} s_{j+n}^{\dagger} t_{j \alpha} t_{j+n \alpha}+\text { H.c. }\right)\right], \\
& i \\
\mathcal{H}_{3}= & -\left(j \leftrightarrow \beta \lambda \sum _ { j , n } \left\{\left[\left(s_{j}^{\dagger} t_{j \alpha}+t_{j \alpha}^{\dagger} s_{j}\right) t_{j+n \beta}^{\dagger} t_{j+n \lambda}+\text { H.c. }\right]\right.\right. \\
& \mathcal{H}_{4}=-\frac{1}{4} \epsilon_{\alpha \beta \lambda} \epsilon_{\alpha \mu \nu} \sum_{j, n} g_{4}(n) t_{j \beta}^{\dagger} t_{j+n \mu}^{\dagger} t_{j+n \nu} t_{j \lambda},
\end{aligned}
$$

where the $\sum_{j}$ runs over the sites of the dimerized lattice, and the $g_{m}(n)$ coefficients read

$$
\begin{aligned}
& g_{2}(n)=\left(2 J^{\prime \prime}-1\right) \delta_{n, 1}-\delta_{n, 2}-\delta_{n, 3}, \\
& g_{4}(n)=\left(2 J^{\prime \prime}+1\right) \delta_{n, 1}+\delta_{n, 2}+\delta_{n, 3} .
\end{aligned}
$$

\section{APPENDIX C: SELF-CONSISTENT EQUATIONS}

Here we derive the self-consistent Eqs. (19) and (20) involved in the harmonic approximation, and Eqs. (28) and (29) related to the HF one. As mentioned in Sec. III, the starting point is the saddle-point conditions: (i) $\partial \bar{E}_{0} / \partial N_{0}=0$ and (ii) $\partial \bar{E}_{0} / \partial \mu=0$.

Let us consider the harmonic case. From the expression (16) for the ground-state energy, we have

$$
\frac{\partial \bar{E}_{0}}{\partial N_{0}}=-\frac{3}{8} J^{\prime} N-\frac{1}{2} \mu N+\frac{3}{2} \sum_{\mathbf{q}}\left(\frac{\partial \omega_{\mathbf{q}}}{\partial N_{0}}-\frac{\partial A_{\mathbf{q}}}{\partial N_{0}}\right) .
$$

Since $\partial A_{\mathbf{q}} / \partial N_{0}=\partial B_{\mathbf{q}} / \partial N_{0}=B_{\mathbf{q}} / N_{0}$, see Eqs. (10) and (11), condition (i) yields Eq. (19). Moreover,

$$
\frac{\partial \bar{E}_{0}}{\partial \mu}=-\frac{1}{2} N\left(N_{0}-1\right)+\frac{3}{2} \sum_{\mathbf{q}}\left(\frac{\partial \omega_{\mathbf{q}}}{\partial \mu}-\frac{\partial A_{\mathbf{q}}}{\partial \mu}\right) .
$$

One can easily see that $\partial A_{\mathbf{q}} / \partial \mu=-1$ and $\partial \omega_{\mathbf{q}} / \partial \mu=$ $-A_{\mathbf{q}} / \omega_{\mathbf{q}}$, and therefore condition (ii) leads to Eq. (20).

Turning to the Hartree-Fock approximation, the equivalent of Eq. (C1) is given here by

$$
\frac{\partial \bar{E}_{0}^{\mathrm{HF}}}{\partial N_{0}}=-\frac{3}{8} J^{\prime} N-\frac{1}{2} \mu N+\frac{3}{2} \sum_{\mathbf{q}}\left(\frac{\partial \bar{\omega}_{\mathbf{q}}}{\partial N_{0}}-\frac{\partial \bar{A}_{\mathbf{q}}}{\partial N_{0}}\right) .
$$

The contribution due to $E_{0}^{\mathrm{HF}}$, Eq. (24), can be neglected since it is $O\left(\bar{v}_{\mathbf{q}}^{2}\right)$ and therefore small compared to the other terms (see the discussion in Sec. IV A). The last term of the above equation can be written as

$$
I=\frac{3}{2} \sum_{\mathbf{q}}\left(-1+\frac{\bar{A}_{\mathbf{q}}}{\bar{\omega}_{\mathbf{q}}}\right) \frac{\partial \bar{A}_{\mathbf{q}}}{\partial N_{0}}-\frac{\bar{B}_{\mathbf{q}}}{\bar{\omega}_{\mathbf{q}}} \frac{\partial \bar{B}_{\mathbf{q}}}{\partial N_{0}}
$$

with

$$
\begin{aligned}
& \frac{\partial \bar{A}_{\mathbf{q}}}{\partial N_{0}}=\frac{B_{\mathbf{q}}}{N_{0}}-\frac{1}{N^{\prime}} \sum_{\mathbf{k}} \gamma_{\mathbf{k}-\mathbf{q}} \frac{\partial}{\partial N_{0}}\left(\frac{\bar{A}_{\mathbf{k}}}{\bar{\omega}_{\mathbf{k}}}\right), \\
& \frac{\partial \bar{B}_{\mathbf{q}}}{\partial N_{0}}=\frac{B_{\mathbf{q}}}{N_{0}}-\frac{1}{N^{\prime}} \sum_{\mathbf{k}} \gamma_{\mathbf{k}-\mathbf{q}} \frac{\partial}{\partial N_{0}}\left(\frac{\bar{B}_{\mathbf{k}}}{\bar{\omega}_{\mathbf{k}}}\right) .
\end{aligned}
$$

Assuming that

$$
\partial \bar{A}_{\mathbf{q}} / \partial N_{0}=\partial \bar{B}_{\mathbf{q}} / \partial N_{0} \approx B_{\mathbf{q}} / N_{0},
$$

and using the expressions (27), we show that $I \approx$ $\left(3 / N_{0}\right) \sum_{\mathbf{q}} B_{\mathbf{q}} \bar{v}_{\mathbf{q}}\left(\bar{v}_{\mathbf{q}}-\bar{u}_{\mathbf{q}}\right)$, and therefore Eq. (28) follows from condition (i). Some words here about the assumption (C4) are in order: we realize that it is a good approximation as long as $\bar{v}_{\mathbf{q}}$ is small. Indeed, using the relations (27), it is possible to show that

$$
\frac{\partial}{\partial N_{0}}\left(\frac{\bar{A}_{\mathbf{k}}}{\bar{\omega}_{\mathbf{k}}}\right)=\frac{4 \bar{u}_{\mathbf{k}}^{2} \bar{v}_{\mathbf{k}}^{2}}{\bar{\omega}_{\mathbf{k}}} \frac{\partial \bar{A}_{\mathbf{q}}}{\partial N_{0}}+\frac{2 \bar{u}_{\mathbf{k}} \bar{v}_{\mathbf{k}}}{\bar{\omega}_{\mathbf{k}}}\left(\bar{v}_{\mathbf{q}}^{2}+\bar{u}_{\mathbf{q}}^{2}\right) \frac{\partial \bar{B}_{\mathbf{k}}}{\partial N_{0}} .
$$

Substituting $(\mathrm{C} 4)$ in the above expression, one can easily see that $\partial \bar{A}_{\mathbf{q}} / \partial N_{0} \approx B_{\mathbf{q}} / N_{0}+O\left(\bar{v}_{\mathbf{k}}\right)$. A similar expression holds for $\partial \bar{B}_{\mathbf{q}} / \partial N_{0}$.

The analog of Eq. (C2) reads

$$
\frac{\partial \bar{E}_{0}^{\mathrm{HF}}}{\partial \mu}=-\frac{1}{2} N\left(N_{0}-1\right)+\frac{3}{2} \sum_{\mathbf{q}}\left(\frac{\partial \bar{\omega}_{\mathbf{q}}}{\partial \mu}-\frac{\partial \bar{A}_{\mathbf{q}}}{\partial \mu}\right) .
$$

Here

$$
\begin{aligned}
\frac{\partial \bar{A}_{\mathbf{q}}}{\partial \mu} & =-1-\frac{1}{N^{\prime}} \sum_{\mathbf{k}} \gamma_{\mathbf{k}-\mathbf{q}} \frac{\partial}{\partial \mu}\left(\frac{\bar{A}_{\mathbf{k}}}{\bar{\omega}_{\mathbf{k}}}\right), \\
\frac{\partial \bar{B}_{\mathbf{q}}}{\partial \mu} & =-\frac{1}{N^{\prime}} \sum_{\mathbf{k}} \gamma_{\mathbf{k}-\mathbf{q}} \frac{\partial}{\partial \mu}\left(\frac{\bar{B}_{\mathbf{k}}}{\bar{\omega}_{\mathbf{k}}}\right) .
\end{aligned}
$$

In order to be consistent with the above approximation, we assume that $\partial \bar{A}_{\mathbf{q}} / \partial \mu \approx-1$ and $\partial \bar{B}_{\mathbf{q}} / \partial \mu \approx 0$, which implies $\partial \bar{\omega}_{\mathbf{q}} / \partial \mu \approx-\bar{A}_{\mathbf{q}} / \bar{\omega}_{\mathbf{q}}$. Notice that this is indeed a reasonable approximation because condition (ii) yields merely the conservation (on average) of the total number of bosons per site of the dimerized lattice, i.e., Eq. (29). 
${ }^{1}$ S. Sachdev, in Quantum Magnetism, Lecture Notes in Physics Vol. 645, edited by U. Schollwöck, J. Richter, D. J. J. Farnell, and R. A. Bishop (Springer, Berlin, 2004); Nat. Phys. 4, 173 (2008).

${ }^{2} \mathrm{G}$. Misguich and C. Lhuillier, in Frustrated Spin Systems, edited by H. T. Diep (World Scientific, Singapore, 2004); C. Lhuillier, e-print arXiv:cond-mat/0502464.

${ }^{3}$ L. Balents, Nature (London) 464, 199 (2010).

${ }^{4}$ P. W. Anderson, Mater. Res. Bull. 8, 153 (1973).

${ }^{5}$ See, e.g., L. Capriotti, A. E. Trumper, and S. Sorella, Phys. Rev. Lett. 82, 3899 (1999); W. Zheng, J. O. Fjaerestad, R. R. P. Singh, R. H. McKenzie, and R. Coldea, Phys. Rev. B 74, 224420 (2006); S. R. White and A. L. Chernyshev, Phys. Rev. Lett. 99, 127004 (2007).

${ }^{6}$ H. Y. Yang, A. M. Lauchli, F. Mila, and K. P. Schmidt, Phys. Rev. Lett. 105, 267204 (2010).

${ }^{7}$ A. E. Trumper, Phys. Rev. B 60, 2987 (1999).

${ }^{8}$ J. Merino, R. H. McKenzie, J. B. Marston, and C. H. Chung, J. Phys.: Condens. Matter 11, 2965 (1999).

${ }^{9}$ Z. Weihong, R. H. McKenzie, and R. P. Singh, Phys. Rev. B 59, 14367 (1999).

${ }^{10}$ R. F. Bishop, P. H. Y. Li, D. J. J. Farnell, and C. E. Campbell, Phys. Rev. B 79, 174405 (2009).

${ }^{11}$ O. A. Starykh and L. Balents, Phys. Rev. Lett. 98, 077205 (2007).

${ }^{12}$ S. Ghamari, C. Kallin, S.-S. Lee, and E. S. Sorensen, Phys. Rev. B 84, 174415 (2011).

${ }^{13}$ A. Weichselbaum and S. R. White, Phys. Rev. B 84, 245130 (2011).

${ }^{14}$ S. Yunoki and S. Sorella, Phys. Rev. B 74, 014408 (2006);

D. Heidarian, S. Sorella, and F. Becca, ibid. 80, 012404 (2009).

${ }^{15}$ J. Reuther and R. Thomale, Phys. Rev. B 83, 024402 (2011).

${ }^{16}$ C. Xu and S. Sachdev, Phys. Rev. B 79, 064405 (2009).

${ }^{17}$ P. Hauke, T. Roscilde, V. Murg, J. I. Cirac, and R. Schmied, New J. Phys. 13, 075017 (2011).

${ }^{18}$ R. Coldea, D. A. Tennant, A. M. Tsvelik, and Z. Tylczynski, Phys. Rev. Lett. 86, 1335 (2001).

${ }^{19}$ T. Ono, H. Tanaka, H. Aruga Katori, F. Ishikawa, H. Mitamura, and T. Goto, Phys. Rev. B 67, 104431 (2003).

${ }^{20}$ Y. Shimizu, K. Miyagawa, K. Kanoda, M. Maesato, and G. Saito, Phys. Rev. Lett. 91, 107001 (2003); Phys. Rev. B 73, 140407(R) (2006).

${ }^{21}$ M. Tamura, A. Nakao, and R. Kato, J. Phys. Soc. Jpn. 75, 093701 (2006)

${ }^{22}$ Y. Shimizu, H. Akimoto, H. Tsujii, A. Tajima, and R. Kato, Phys. Rev. Lett. 99, 256403 (2007).
${ }^{23}$ T. Itou, A. Oyamada, S. Maegawa, K. Kubo, H. M. Yamamoto, and R. Kato, Phys. Rev. B 79, 174517 (2009).

${ }^{24}$ M. Tamura and R. Kato, Sci. Technol. Adv. Mater. 10, 024304 (2009).

${ }^{25}$ T. Itou, A. Oyamada, S. Maegawa, M. Tamura, and R. Kato, Phys. Rev. B 77, 104413 (2008).

${ }^{26}$ The dimerization pattern in Fig. 1 appears different from the one proposed for $\mathrm{EtMe}_{3} \mathrm{P}\left[\mathrm{Pd}(\mathrm{dmit})_{2}\right]_{2}$ in Ref. 21, but we note that the undistorted phase of $\mathrm{EtMe}_{3} \mathrm{P}\left[\mathrm{Pd}(\mathrm{dmit})_{2}\right]_{2}$ is very close to the isotropic point with the three exchange couplings being equal within $6 \%$, such that the dimer states are nearly degenerate. One of our motivations to choose the dimer pattern of Fig. 1 was to make contact with the staggered dimerized square-lattice model of Refs. 33 and 34.

${ }^{27}$ S. Sachdev and R. N. Bhatt, Phys. Rev. B 41, 9323 (1990).

${ }^{28}$ A. Dutta, B. K. Chakrabarti, and J. K. Bhattacharjee, Phys. Rev. B 55, 5619 (1997)

${ }^{29}$ R. Ramazashvili, Phys. Rev. B 60, 7314 (1999).

${ }^{30}$ A. Kolezhuk and S. Sachdev, Phys. Rev. Lett. 96, 087203 (2006).

${ }^{31}$ M. E. Zhitomirsky, Phys. Rev. B 73, 100404 (2006).

${ }^{32}$ T. Giamarchi, C. Rüegg, and O. Tchernyshyov, Nat. Phys. 4, 198 (2008).

${ }^{33}$ S. Wenzel, L. Bogacz, and W. Janke, Phys. Rev. Lett. 101, 127202 (2008).

${ }^{34}$ L. Fritz, R. L. Doretto, S. Wessel, S. Wenzel, S. Burdin, and M. Vojta, Phys. Rev. B 83, 174416 (2011).

${ }^{35}$ A. L. Chernyshev and M. E. Zhitomirsky, Phys. Rev. B 79, 144416 (2009).

${ }^{36}$ A. V. Chubukov and D. K. Morr, Phys. Rev. B 52, 3521 (1995).

${ }^{37}$ V. N. Kotov, O. Sushkov, Z. Weihong, and J. Oitmaa, Phys. Rev. Lett. 80, 5790 (1998).

${ }^{38}$ V. N. Kotov, J. Oitmaa, O. P. Sushkov, and Z. Weihong, Phys. Rev. B 60, 14613 (1999).

${ }^{39}$ P. V. Shevchenko, V. N. Kotov, and O. P. Sushkov, Phys. Rev. B 60, 3305 (1999).

${ }^{40}$ Strictly, the cubic interaction generates both normal and anomalous self-energies, which then yield a $2 \times 2$ matrix structure of the Dyson equation. However, in the $b$ basis, the anomalous self-energy is small provided that $\bar{v}_{\mathbf{k}}$ is small: For selected parameters, we have checked that including the anomalous self-energy in the on-shell approximation changes the triplon energy by less than $1 \%$.

${ }^{41}$ L. Wang and S. G. Chung, e-print arXiv:1110.0377. 